\title{
Liquiritin suppresses UVB-induced skin injury through prevention of inflammation, oxidative stress and apoptosis through the TLR4/MyD88/NF-кB and MAPK/caspase signaling pathways
}

\author{
XIAO-QING LI, LI-MIN CAI, JING LIU, YAN-LI MA, YING-HUI KONG, HE LI and MING JIANG \\ Department of Dermatology, The Affiliated Huaian No. 1 People's Hospital of Nanjing Medical University, \\ Huai'an, Jiangsu 223300, P.R. China
}

Received March 19, 2017; Accepted May 9, 2018

DOI: $10.3892 /$ ijmm.2018.3720

\begin{abstract}
Solar ultraviolet B (UVB) radiation is known to trigger inflammation, oxidative stress and apoptotic responses through various signaling pathways, which eventually lead to skin cancer. The present study investigated whether liquiritin suppresses UVB-induced skin injury in vivo and in vitro using SKH-1 hairless mice and HACAT cells, respectively. The animals were exposed to UVB irradiation $\left(180 \mathrm{~mJ} / \mathrm{cm}^{2}\right)$ for $20 \mathrm{~min}$, followed by liquiritin treatment. The findings indicated that UVB exposure resulted in the excessive release of pro-inflammatory cytokines, including interleukin (IL)- $1 \beta$, tumor necrosis factor (TNF)- $\alpha$, IL-18, IL- 6 and cyclooxygenase $(\mathrm{COX}) 2$, which were dependent on the toll-like receptor (TLR)4/myeloid differentiation factor 88 (MyD88)/nuclear factor- $\kappa \mathrm{B}(\mathrm{NF}-\kappa \mathrm{B})$ signaling pathway. Oxidative stress was also observed, evidenced by reduced antioxidants and elevated oxidants. Apoptosis, examined using terminal deoxynucleotidyl transferase dUTP nick end labeling and crystal violet staining, suggested that UVB irradiation caused cell death in vivo and in vitro, which was closely associated with p38/c-Jun N-terminal kinase and caspase activity. Of note, liquiritin treatment in mice and cells exposed to UVB showed reduced inflammatory response, oxidative stress and apoptosis through inhibiting the activation of TLR4/MyD88/NF- $\mathrm{KB}$ mitogen-activated protein kinases and caspase pathways, and downregulating the release of oxidants. Overall, the data revealed that liquiritin may be a useful compound against UVB-induced skin injury.
\end{abstract}

Correspondence to: Dr Xiao-Qing Li, Department of Dermatology, The Affiliated Huaian No. 1 People's Hospital of Nanjing Medical University, 6 Beijing Road West, Huai'an, Jiangsu 223300, P.R. China E-mail: lixiaoqingha@foxmail.com

Key words: ultraviolet $\mathrm{B}$ radiation, liquiritin, inflammation, oxidative stress, apoptosis

\section{Introduction}

Skin is characterized as the first defense against harmful biological, physical and environmental pollutants, including ultraviolet (UV) irradiation (1). Excessive UVB exposure has been reported to disrupt biological macromolecules, including proteins, lipids and nucleic acids, leading to various disorders of skin and ultimately to skin cancer $(2,3)$. Additionally, solar UVB irradiation can result in sunburn, proliferation, oxidative stress, apoptosis, oxidative stress and erythema, which are associated with various signaling pathways, including the toll-like receptor (TLR) 4 /nuclear factor- $\mathrm{KB}$ (NF- $\mathrm{kB}$ ) and mitogen-activate protein kinases (MAPKs) (4). Pro-inflammatory cytokines, including interleukin (IL)-1 $\beta$, tumor necrosis factor (TNF)- $\alpha$, IL-18, IL-6 and cyclooxygenase (COX)2, have been suggested to be crucial in the progression of inflammatory responses $(5,6)$. UVB, as previously reported, can lead to the high release of COX2. COX2 is involved in the inflammatory response, cell survival and proliferation (7). The secretion of pro-inflammatory cytokines is linked to activation of the TLR4/NF- $\mathrm{KB}$ signaling pathway. TLRs are important in initiating the inflammatory response. Several stimuli can activate TLR4. Once activated, myeloid differentiation factor 88 (MyD88), a key adapter protein for TLR4, leads to the direct activation of NF- $\mathrm{KB}$ and the subsequent induction of pro-inflammatory cytokines, including TNF- $\alpha$, IL- $1 \beta$ and IL- 6 , which contribute to the inflammatory response $(8,9)$. Drugs inhibiting the activity of the TLR inflammatory system afford potential beneficial effects for various tissues or organs, including the skin, as do transgenic methods of inhibiting TLR4/NF-kB-related genes (10). Reactive oxygen species (ROS), reflecting levels of oxidative stress, are closely associated with a variety of biomolecules, leading to damage of biological structures, and contributing to cellular injury and ultimately tissue destruction. The skin has evolved effective mechanisms for the protection from ROS damage, which involves ROS detoxification and DNA repair (11). Therefore, the excessive production of ROS disrupts molecular balances, promoting the pathogenesis of inflammatory skin diseases, which identifies a target for drug investigations.

MAPKs, including p38, extracellular signal-regulated kinase (ERK)1/2 and c-Jun N-terminal kinase (JNK), are 
important in regulating apoptosis (12). P38 and JNK are often activated in human tumors, and are associated with cell proliferation, migration and metastasis. p38-MAPK has also been described as a tumor suppressor, particularly under situations of stress (13). The p38-MAPK pathway can be regulated by transcriptional and post-transcriptional mechanisms to affect cell death signaling, and pro- and anti-apoptotic B-cell lymphoma 2 proteins (14). Subsequently, the caspase signaling pathway is altered, contributing to apoptosis (15). Therefore, targeting the MAPK-regulated signaling pathway may be of potential value in UVB-induced apoptosis.

Liquiritin, a major constituent of Glycyrrhiza Radix, has various pharmacological activities (16). A previous study reported that liquiritin may exert neuroprotective effects in cerebral ischemia/reperfusion-induced brain damage through antioxidant and anti-apoptotic mechanisms (17). As reported previously, liquiritin ameliorates tissue injury through suppressing MAPK and TLR4/MyD88 signaling pathways, which reduces the inflammatory response (18). In addition, liquiritin suppresses the progression of breast cancer, with a reliance on the regulation of ROS (19). Therefore, the present study hypothesized that liquiritin may be effective in preventing skin injury induced by UVB, which is associated with inflammation, ROS and apoptosis. However, until now, the role of liquiritin in this regard remains to be elucidated. The present study indicated for the first time, to the best of our knowledge, that liquiritin can be used as an effective compound to protect the skin from damage by UVB in vivo and in vitro. Liquiritin pre-treatment suppressed UVB-induced inflammation, oxidative stress and apoptosis via inactivating the TLR4/MyD88/NF- $\kappa$ B and MAPK/caspase signaling pathways.

\section{Materials and methods}

Animals and treatments. A total of 110 female, 6-8-week-old SKH-1 hairless mice (18-20 g) were purchased from Shanghai Laboratory Animal Research Center (Shanghai, China). The mice were acclimatized for 1 week prior to experiments in the specific pathogen-free conditions in static microisolator cages with tap water ad libitum, and were maintained under standard conditions of a $12 \mathrm{~h}$ dark/12 h light cycle (8:00 a.m. to $8: 00$ p.m.) at a temperature of $23 \pm 2{ }^{\circ} \mathrm{C}$ and relative humidity of $50 \pm 5 \%$. All animal experiments were performed following the Guide for the Care and Use of Laboratory Animals, issued by the National Institutes of Health in 1996 and approved by Huai'an First People's Hospital, Nanjing Medical University (Nanjing, China). The care and handling of mice were in accordance with the ethical guidelines of Huai'an First People's Hospital. The SKH-1 hairless mice were randomly divided into four groups with 15 mice in each group cage. The mice were exposed to UVB lamps (GL20SE; Sankyo Denki Co., Ltd., Hiratsuka, Japan) equipped with a controller to modulate UV dosage, at a distance of $20 \mathrm{~cm}$ between the target skin and the light source. The treatment groups, containing 15 animals in each group, were as follows: Group 1, untreated animals (Con); Group 2, animals irradiated with UVB only (UVB); Group 3, UVB irradiation with application of liquiritin $(10 \mathrm{mg} / \mathrm{kg})$ by gavage (UVB+10); Group 4, UVB irradiation with application of liquiritin $(20 \mathrm{mg} / \mathrm{kg})$ by gavage $(\mathrm{UVB}+20)$ following UVB $\left(50 \mathrm{~mJ} / \mathrm{cm}^{2}\right)$ for $30 \mathrm{~min}$. The murine skin exposure was then performed at $50 \mathrm{~mJ} / \mathrm{cm}^{2}$ of UVB, three times per week for a consecutive 10-week period. Following another 7 weeks of exposure with or without liquiritin treatment, serum samples of animals were collected through eye bleeding $24 \mathrm{~h}$ following the final UVB irradiation, and the dorsal skin tissues from mice were excised and collected for following analysis (Fig. 1A). In addition, 30 mice were treated with liquiritin (25, 50 and $100 \mathrm{mg} / \mathrm{kg}$ ) in the absence of UVB irradiation to investigate the hepatotoxicity of liquiritin in mice. The mice treated with liquiritin were pre-treated at the described concentrations for 2 weeks. The liquiritin (purity $\geq 98 \%$ ) used in the present study was purchased from the Chinese Institute for the Control of Pharmaceutical and Biological Products (Beijing, China).

Cell culture and treatments. Human epidermal cells (HACAT) and the L02 human liver normal cell line were purchased from the American Type Culture Collection (Manassas, VA, USA) and Nanjing KeyGEN Biotech Co., Ltd. (Nanjing, China), respectively. The cells were cultured in DMEM supplemented with 10\% FBS (Invitrogen; Thermo Fisher Scientific, Inc., Waltham, MA, USA), penicillin $(100 \mathrm{U} / \mathrm{ml})$ and streptomycin $(100 \mu \mathrm{g} / \mathrm{ml})$ at $37^{\circ} \mathrm{C}$ in an atmosphere of $5 \% \mathrm{CO}_{2}$. The HACAT cells were cultured until $80 \%$ confluence and then pretreated with various concentrations of liquiritin (40 and $80 \mu \mathrm{M})$. Following incubation for $2 \mathrm{~h}$, the culture medium was replaced with $1.5 \mathrm{ml}$ of phosphate-buffered saline (PBS). Subsequently, the HACAT cells were exposed to UVB $\left(15 \mathrm{~mJ} / \mathrm{cm}^{2}\right)$ light at a $312 \mathrm{~nm}$ light source for $1 \mathrm{~h}$. Following UVB-exposure, the cells were treated with various concentrations of liquiritin (40 and $80 \mu \mathrm{M}$ ) in serum-free medium for another $22 \mathrm{~h}$. The cells were then harvested for further examination.

Cell viability analysis. The HACAT human epidermal cell line and L02 human liver normal cell line were initially seeded in 96-well plates at a density of $2 \times 10^{4}$ cells/well, respectively, prior to incubation for $24 \mathrm{~h}$ at $37^{\circ} \mathrm{C}$. The cell culture media were then replaced with complete media containing the indicated concentrations $(0,1.25,2.5,5,10,20,40,80$ and $160 \mu \mathrm{M})$ of liquiritin, prior to incubation for the indicated duration $(0,6,12,24,36,48,72$ and $96 \mathrm{~h})$. Following incubation as indicated, $10 \mu \mathrm{l}$ of MTT (Nanjing KeyGEN Biotech, Co., Ltd.) was administrated to cells, followed by incubation for $4 \mathrm{~h}$ at $37^{\circ} \mathrm{C}$ according to the manufacturer's protocol. Finally, the absorbance was read at $570 \mathrm{~nm}$ on a microplate reader. The cell viability (\%) was evaluated as the ratio of surviving cells.

Crystal violet staining. Following the various treatments, the HACAT cells were harvested and a Crystal Violet Staining Solution kit (Beyotime Institute of Biotechnology, Haimen, China) was used to analyze the HACAT cell proliferation, according to the manufacturer's protocol.

Analysis of chemical indicators. The activities of enzymatic antioxidants in serum and skin tissues, including superoxide dismutase (SOD) and catalase (CAT), were analyzed using an SOD assay kit (Nanjing Jiancheng Bioengineering Institute, Nanjing, China) and a CAT assay kit (Nanjing Jiancheng Bioengineering Institute), respectively. The level of malondialdehyde (MDA) in skin tissues was measured using an MDA assay (Nanjing Jiancheng Bioengineering Institute) 
A
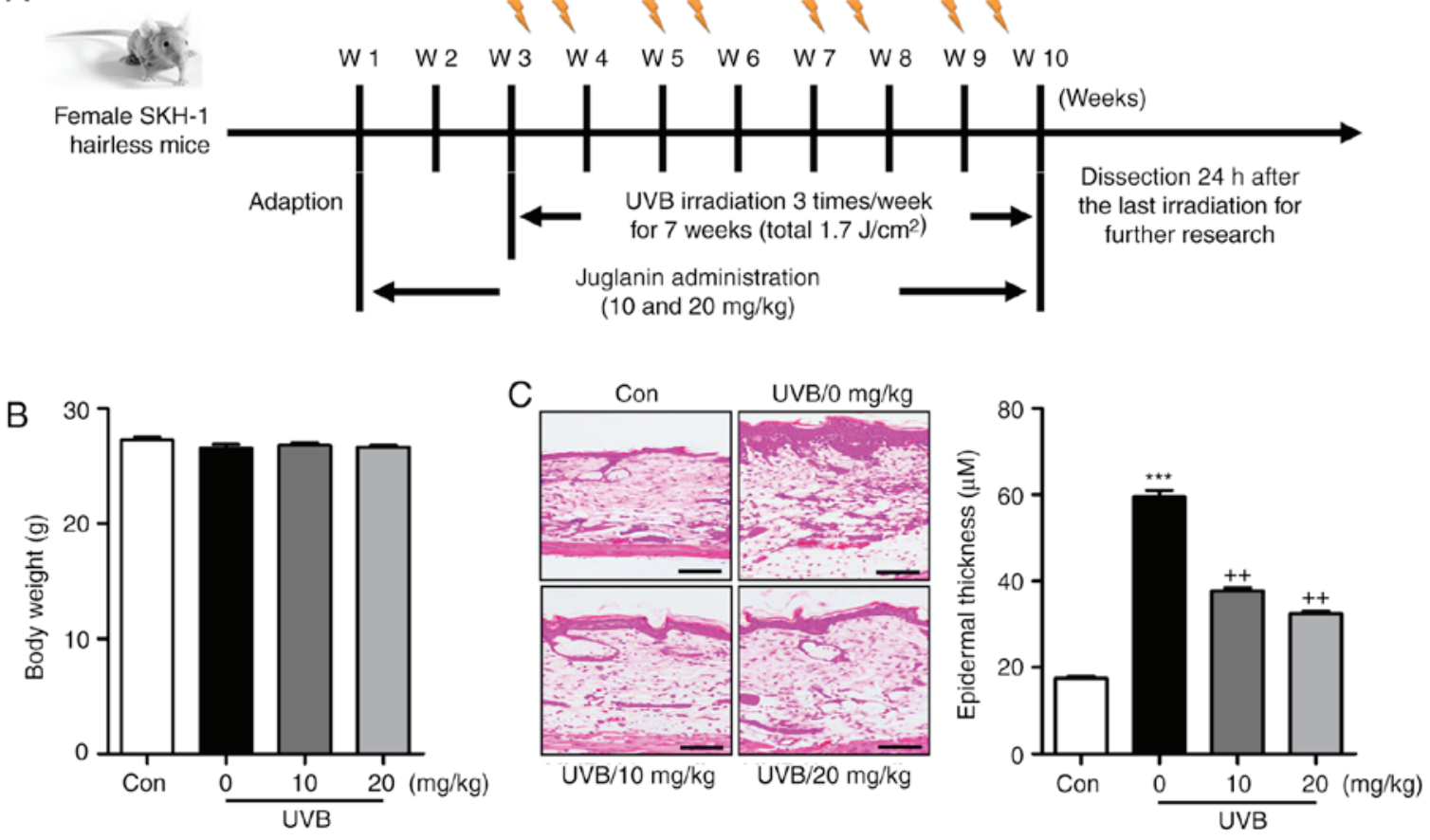

Figure 1. Liquiritin improves UVB-induced skin injury in mice. (A) Experimental design. (B) Body weights of mice from each group were measured. (C) Hematoxylin and eosin staining was used to calculate the extent of skin injury. Representative images and quantification are shown. Scale bar, $100 \mu \mathrm{m}$. Data are presented as the mean \pm standard error of the mean $(n=10) .{ }^{* * * *} \mathrm{P}<0.001$, compared with the Con group. ${ }^{++} \mathrm{P}<0.01$ compared with the UVB-only (no liquiritin) group. UVB, ultraviolet B; W, week; Con, control.

kit according to the manufacturer's protocol. The levels of aspartate aminotransferase (AST) and alanine aminotransferase (ALT) in serum were calculated to determine the hepatotoxicity using specific kits purchased from Nanjing Jiancheng Bioengineering Institute according to the manufacturer's protocol. The levels of $\mathrm{H}_{2} \mathrm{O}_{2}$ in the skin tissue sections were calculated using a hydrogen peroxide assay kit (Nanjing Jiancheng Bioengineering Institute). $\mathrm{O}_{2}^{-}$in the skin tissue samples was evaluated using the lucigenin chemiluminescence method. Briefly, the mice skin tissue samples were weighed and homogenized in homogenization buffer with HEPES and EDTA. Following centrifugation at $1,000 \mathrm{x}$ g for $10 \mathrm{~min}$ at $4^{\circ} \mathrm{C}$, an aliquot $(200 \mu \mathrm{l})$ of the supernatant was further incubated with $5 \mu \mathrm{M}$ lucigenin in Krebs-HEPES buffer. The light emission was measured with a Tecan Infinite 200 reader. The specificity for $\mathrm{O}_{2}^{-}$was evaluated by adding SOD $(350 \mathrm{U} / \mathrm{ml})$ into the incubation medium. Protein content was then measured with a BCA Protein Quantitative Analysis kit (Thermo Fisher Scientific, Inc.).

ELISA methods. The skin samples were frozen in liquid nitrogen and crushed into a powder with a multibead shocker. The powder was then dissolved in cell lysis buffer for western blot analysis and immunoprecipiation (Beyotime Institute of Biotechnology) with protease inhibitor cocktail. The skin extract was prepared by centrifugation at $12,000 \mathrm{x}$ g for $10 \mathrm{~min}$ at $4^{\circ} \mathrm{C}$, and the supernatant was retained for further analysis. The sample protein concentrations were calculated using a BCA protein assay kit (Thermo Fisher Scientific, Inc.). The protein levels of IL- $1 \beta$, TNF- $\alpha$, IL-18, IL- 6 and COX 2 were assessed using respective mouse ELISA kits (R\&D Systems,
Inc., Minneapolis, MN, USA) according to the manufacturer's protocol. Finally, the absorbance was read at $450 \mathrm{~nm}$ on a microplate reader.

Analyses of transepidermal water loss (TEWL), hydration and elasticity. TEWL $\left(\mathrm{g} / \mathrm{m}^{2} / \mathrm{h}\right)$ is a marker of epidermal skin barrier function, and was measured with a Tewameter ${ }^{\mathrm{TM}} 300$ (Courage and Khazaka Electronics GmbH, Köln, Germany). In brief, under isoflurane anesthesia, a medical adhesive tape (Honsmed, Shanghai, China) was attached to the mouse skin under a gentle pressure, following which it was removed. The mice were tape-stripped five times. TEWL was then recorded with the Tewameter ${ }^{\text {TM }} 300$ device. The moisture levels of the stratum corneum were evaluated through a CM825 corneometer (Courage and Khazaka Electronics $\mathrm{GmbH}$ ) and the skin elasticity, indicated by the parameter F3, was assessed with a cutometer of DUAL MPA580 (Courage and Khazaka Electronics $\mathrm{GmbH}$ ) according to the manufacturer's protocols.

Terminal deoxynucleotidyl transferase dUTP nick end labeling (TUNEL) assays. The TUNEL assay (BioVision, Inc., Milpitas, CA, USA) was used to measure the apoptotic cells according to the manufacturer's protocol. The skin sections were treated with proteinase $\mathrm{K}(20 \mu \mathrm{g} / \mathrm{ml})$ at $37^{\circ} \mathrm{C}$ for $15 \mathrm{~min}$, followed by incubation with TUNEL reaction mixture at $37^{\circ} \mathrm{C}$ for $1 \mathrm{~h}$. Subsequently, each section was exposed to an antibody solution (anti-BrdU monoclonal antibody; 1:250; cat. no. ab6326; Abcam, Cambridge, MA, USA) for $30 \mathrm{~min}$ at room temperature. The TUNEL-stained cells were observed using a light microscope (Nikon, Tokyo, Japan) equipped with an ocular micrometer in five randomly selected fields 
per section. The level of apoptosis (\%) was calculated as the average number of stained cells in each field divided by the total number of cells.

Western blot analysis. The HACAT cells or mice skin tissue samples were lysed using RIPA buffer containing a 1:100 dilution of protease inhibitor and phosphatase inhibitor (Baomanbio, Shanghai, China). The lysates were then centrifuged at $15,000 \mathrm{x} \mathrm{g}$ for $15 \mathrm{~min}$ at $4^{\circ} \mathrm{C}$ to collect the supernatant. The protein concentrations were evaluated using a BCA protein assay (Thermo Fisher Scientific, Inc.), and equal protein quantities $(40 \mu \mathrm{g})$ were separated using $10 \%$ SDS-PAGE. The proteins were then electrophoretically transferred onto polyvinylidene difluoride membranes (EMD Millipore, Billerica, MA, USA), and then incubated with Tris-buffered saline containing $0.1 \%$ Tween-20 with 5\% skim milk (BD Biosciences, Franklin Lakes, NJ, USA) at room temperature for $2 \mathrm{~h}$. Primary antibodies dissolved in blocking buffer were used to detect the target protein blots at $4^{\circ} \mathrm{C}$ overnight. The following antibodies were used: Rabbit anti-phosphorylated (p-)P38 (1:1,000; cat. no. 4511; Cell Signaling Technology, Inc., Danvers, MA, USA), rabbit anti-P38 (1:1,000; cat. no. 8690; Cell Signaling Technology, Inc.), rabbit anti-poly(ADP-ribose) polymerase (PARP) (1:1,000; cat. no. 9532; Cell Signaling Technology, Inc.), rabbit anti-TLR4 (1:1,000; cat. no. 14358; Cell Signaling Technology, Inc.), rabbit anti-MyD88 (1:1,000; cat. no. 4283; Cell Signaling Technology, Inc.), and rabbit anti-p-inhibitor of NF- $\kappa$ B kinase (IKK) $\alpha$ (1:1,000; cat. no. 2682; Cell Signaling Technology, Inc.), rabbit anti-inhibitor of NF- $\mathrm{NB}(\mathrm{I} \kappa \mathrm{B}) \alpha$ (1:500; cat. no. 4814; Cell Signaling Technology, Inc.), rabbit anti-p-JNK (1:1,000; cat. no. ab4821; Abcam), rabbit anti-JNK (1:1,000; cat. no. ab131499; Abcam), rabbit anti-caspase-3 (1:1,000; cat. no. ab13847; Abcam), rabbit anti-xanthine oxidase (XO) (1:1,000; cat. no. ab133268; Abcam), rabbit anti-inducible nitric oxide synthase (iNOS) $(1: 1,000$; cat. no. ab15323; Abcam), rabbit anti-NOX2 (1:1,000; cat. no. ab80508; Abcam), rabbit anti-NOX4 (1:1,000; cat. no. ab216654; Abcam), rabbit anti-NF-кB (1:1,000; cat. no. ab207297; Abcam), rabbit anti-p-NF-кB (1:1,000; cat. no. ab86299; Abcam), mouse anti-caspase-9 (1:1,000; cat. no. ab32539; Abcam) and anti-GAPDH (1:1,000; cat. no. ab8245; Abcam). Then, the membranes were washed with tris-buffered saline with Tween-20 (1\%) three times, followed by incubation with a goat-anti rabbit horseradish peroxidase-conjugated secondary antibody (1:2,500; cat. no. ab6721; Abcam) at room temperature for $2 \mathrm{~h}$. The bands on the membrane were analyzed using chemiluminescence with Pierce ECL Western Blotting Substrate reagents (Thermo Fisher Scientific, Inc.). Protein expression levels were assigned a grey value using ImageJ 1.38 software (National Institutes of Health, Bethesda, MD, USA) and standardized to the housekeeping gene GAPDH and expressed as a fold of the control. All experiments were performed in triplicate and performed three times independently.

Reverse transcription-quantitative polymerase chain reaction (RT-qPCR) analysis. Total RNA from the cells and skin tissue samples was extracted using TRIzol reagent (Invitrogen; Thermo Fisher Scientific, Inc.) according to the manufacturer's protocol. Subsequently, RNA were quantified and subjected to reverse transcription to prepare cDNA using a RevertAid
Table I. Sequences of primers used in reverse transcription-quantitative polymerase chain reaction analysis.

Gene $\quad$ Primer sequence (5'-3')

Mouse-TNF- $\alpha$

F: AGATATTACAGTGCCATGGTT R: ACGAGTAGAGATCTCGAG

Mouse-COX2

F: CCAGACTTGGCTACCTCGTG R: CTGACTTAGTCGATAATTCT

Mouse-SOD1 F: GCCATTCCTGTCTCGTGGAG R: TAATGTATTGATCCTTGATTAT

Mouse-IL-18 F: AGTAAGTGGCATTACCGAC R: ACCAACGCAACAGTCTGCAG

Mouse-IL-6

F: GTCAAGACCACAGCTAGC R: CTGGCGACTACTAGTAGATA

Mouse-IL-1 $\beta$ F: ACTCGAGAGGAATCCTTACGA R: CACTCTCGTGACTCGCTA

Mouse-CAT

F: CCTCGTTCACACTTCGTGTA R: GGAAGCACTCCAGTGCAGC

Mouse-Nrf2

F: CTACAGACCAATGCCTGAC R: ACGTAACTGTGCCATGGGGA

Mouse-SOD2 F: AGGCCGTGAGAGCTTGTGTA R: CGGCAGTAAGTGCCCTCTAC

Mouse-GAPDH F: GCGAGCTGAGACACTCTAG R: TAGGCATGCACCTCTGTTCA

Human-TNF- $\alpha$ F: ATATGTGCTGGTCATACTCAT R: AGAGATCGGCGTCAGATGA

Human-COX2 F: CCTTGCAGAGCTACCGAGT R: GTGACTTAGTATATTCATCC

Human-SOD1 F: GTCCTACGGTCTCGTGAGATAT R: GTTAATATTGGTAGTTCTCTG

Human-IL-18 F: AGCATTACCGACCTATTCCT R: AGCACCCAACGCAACAAGTG

Human-IL-6 F: CAGTAGAAGACCAAGTACAT R: CTGGCGACTACTTAGCTATAA

Human-IL-1 $\beta$ F: AATTGAGAGCTCGGATCCGT R: CTGACGCTATCACGTAGAGCA

Human-CAT F: CTCACTACGTCGTGTATTCCCT R: GTGAAGCACTCCAGTGCCAG

Human-Nrf2 F: CTCCAATGCCTGATCGCGCT R: AGGCCATGGCAAGTGTGAT

Human-SOD2

F: CGTGAGGCGAGCTACAGAA R: GGTAAGTTACGCTCCAGCCTC

Human-GAPDH F: GGACCGAGTACTGCATCAGCTA R: TCCTCGTGAAGGTTGACTGCGA

TNF- $\alpha$, tumor necrosis factor- $\alpha$; COX2, cyclooxygenase 2; SOD, superoxide dismutase; IL, interleukin; CAT, catalase; Nrf2, nuclear factor erythroid 2-related factor 2; F, forward; R, reverse.

First Strand cDNA Synthesis kit (Thermo Fisher Scientific, Inc.). For analysis, single-stranded cDNA was prepared 

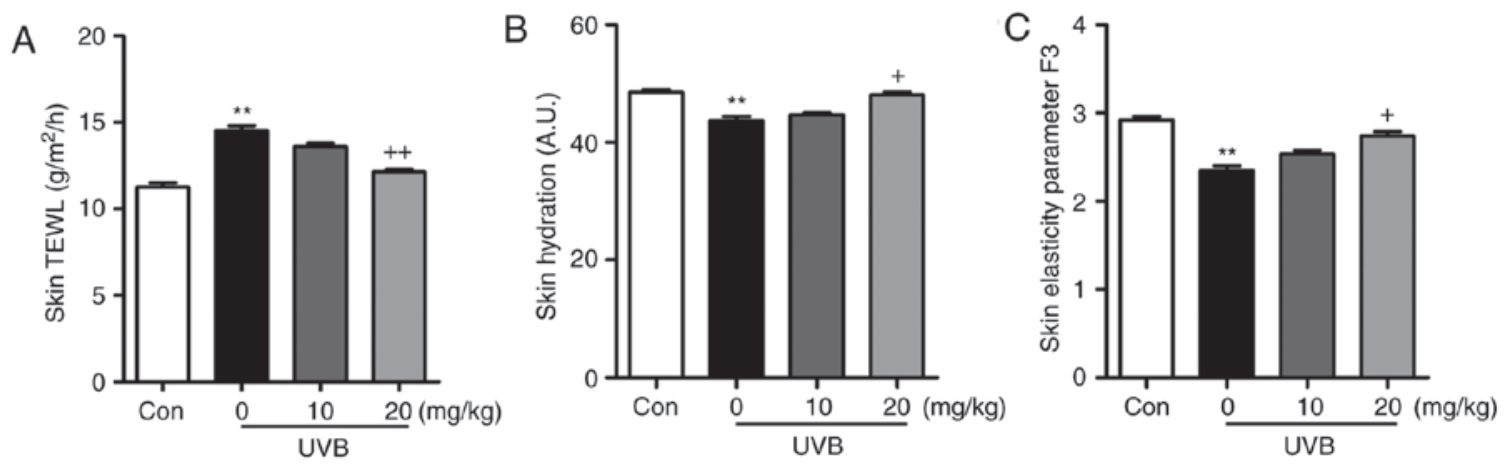

Figure 2. Effects of liquiritin on skin barrier function in UVB-induced mice. (A) Skin TEWL, (B) skin hydration and (C) skin elasticity parameter F3 were measured to examine the role of liquiritin in the regulation of skin induced by UVB. Data are presented as the mean \pm standard error of the mean $(n=10)$. ${ }^{* *} \mathrm{P}<0.01$, compared with the Con group. ${ }^{+} \mathrm{P}<0.05$ and ${ }^{++} \mathrm{P}<0.01$, compared with the UVB-only group. UVB, ultraviolet $\mathrm{B}$; TEWL, transepidermal water loss; Con, control.

from RNA by reverse transcription using oligo(dT) primers (SunShine Biotechnology International Co., Ltd., Somerset, NJ, USA). The RT reaction contained RNA samples including $2 \mu \mathrm{g}$ purified total RNA, $2 \mu 110 \mu \mathrm{M}$ oligo(dT) primers, $5 \mu 1$ 5xRT buffer (Promega Corporation, Madison, WI, USA), $1 \mu 140 \mathrm{U} / \mu 1$ MultiScribe reverse transcriptase (Promega Corporation), $1.25 \mu \mathrm{l} 10 \mathrm{mM}$ dNTPs, $0.625 \mu \mathrm{l} 40 \mathrm{U} / \mu \mathrm{l}$ RNase inhibitor (SunShine Biotechnology International Co., Ltd.), and $25 \mu 1 \mathrm{H}_{2} \mathrm{O}$-diethyl pyrocarbonate. qPCR was performed on a CFX96 Real-Time system (Bio-Rad Laboratories, Inc., Hercules, CA, USA). The sequences of primers were commercially synthesized and the sequences of the primers are listed in Table I. The reaction conditions were as follows: denaturation at $95^{\circ} \mathrm{C}$ for $10 \mathrm{~min}$, followed by 40 cycles of amplification and quantification at $95^{\circ} \mathrm{C}$ for $30 \mathrm{sec}, 60^{\circ} \mathrm{C}$ for $30 \mathrm{sec}$ and $72^{\circ} \mathrm{C}$ for $1 \mathrm{~min}$. The melt curve conditions were as follows: $95^{\circ} \mathrm{C}$ for $15 \mathrm{sec}, 60^{\circ} \mathrm{C}$ for $15 \mathrm{sec}$ and $95^{\circ} \mathrm{C}$ for $15 \mathrm{sec}$. The mRNA expression normalized to the expression of the housekeeper GAPDH was measured using the $2^{-\Delta \Delta C q}$ method (20).

Immunohistochemical analysis. The skin tissues obtained from the mice were fixed, embedded in paraffin blocks and cut into $3-\mu \mathrm{m}$ thick sections. The skin sections were deparaffinized and stained with hematoxylin and eosin (H\&E) staining. The thickness of the skin epidermis was assessed using Magnuspro software (Magnus pro 3.0 software; Olympus Corporation, Tokyo, Japan). The epidermal thickness of the H\&E-stained sections was assessed using Image-J software $(1.47 \mathrm{v}$ version; National Institutes of Health, Bethesda, MD, USA). For immunohistochemical images, the skin tissue sections were then exposed to $\mathrm{HCl}(3.5 \mathrm{M})$ for $20 \mathrm{~min}$ at room temperature and washed using PBS three times. Subsequently, the skin tissue sections were treated with peroxidase $(0.3 \%)$ to block endogenous peroxidase activity. The tissue sections were then incubated with normal goat serum (5\%; cat. no. 5425; Cell Signaling Technology, Inc.) for $30 \mathrm{~min}$, followed by incubation with primary antibodies TLR4 and NOX-2; Abcam,Cambridge, MA, USA) at 1:100 dilution for $2 \mathrm{~h}$ at room temperature. The sections were then incubated with HRP-conjugated compact polymer systems. Diaminobenzidine (Chem Service, West Chester, PA, USA) was used as the chromogen according to the manufacturer's protocol.
Statistical analysis. Data are expressed as the mean \pm standard error of the mean. The statistical analysis was performed using one-way analysis of variance with Tukey's post-hoc test (on GraphPad Prism 6.01; GraphPad Software, Inc., La Jolla, CA, USA). $\mathrm{P}<0.05$ was considered to indicate a statistically significant difference.

\section{Results}

Liquiritin improves UVB-induced skin injury in mice. As shown in Fig. 1B, the present study first examined the body weights of mice treated under different conditions to assess the role of liquiritin in mice. The results indicated there was no significant difference in the body weight of mice treated with various concentrations of liquiritin with UVB exposure, indicating that the doses used in the present study, at least in part, were safe for the animals. Subsequently, the H\&E staining suggested that the epidermal thickness of the skin of the mice was higher in the UVB-treated mice in absence of liquiritin, compared with that of mice in the Con group, and was reduced in the mice exposed to liquiritin treatments, compared with that in the UVB-only group of mice (Fig. 1C). Furthermore, the skin TEWL, skin hydration and skin elasticity parameter $\mathrm{F} 3$ were highly induced in the UVB-irradiated mice, which were comparable to those in the Con group. Following liquiritin treatments, these indicators were reduced (Fig. 2A-C). These data indicated that liquiritin had a potential role in ameliorating skin injury in mice exposed to UVB.

Liquiritin reduces the inflammatory response in UVB-induced skin in mice. The inflammatory response has been characterized as an essential factor in accelerating the progression of various diseases, including liver injury, heart fibrosis and several types of cancer (21). In the present study, the levels of pro-inflammatory cytokines in the skin tissue samples were measured and showed that IL-1 $\beta$ (Fig. 3A), TNF- $\alpha$ (Fig. 3B), IL-18 (Fig. 3C), IL-6 (Fig. 3D) and COX2 (Fig. 3E) were released at high levels in the skin of mice exposed to UVB, which were significantly reduced by liquiritin administration in a dose-dependent manner. The results of the RT-qPCR analysis also indicated that the mRNA levels of IL- $1 \beta$, TNF- $\alpha$, IL-18, IL-6 and COX2 were expressed at high levels in the UVB-treated mice. Similar results were observed in the 

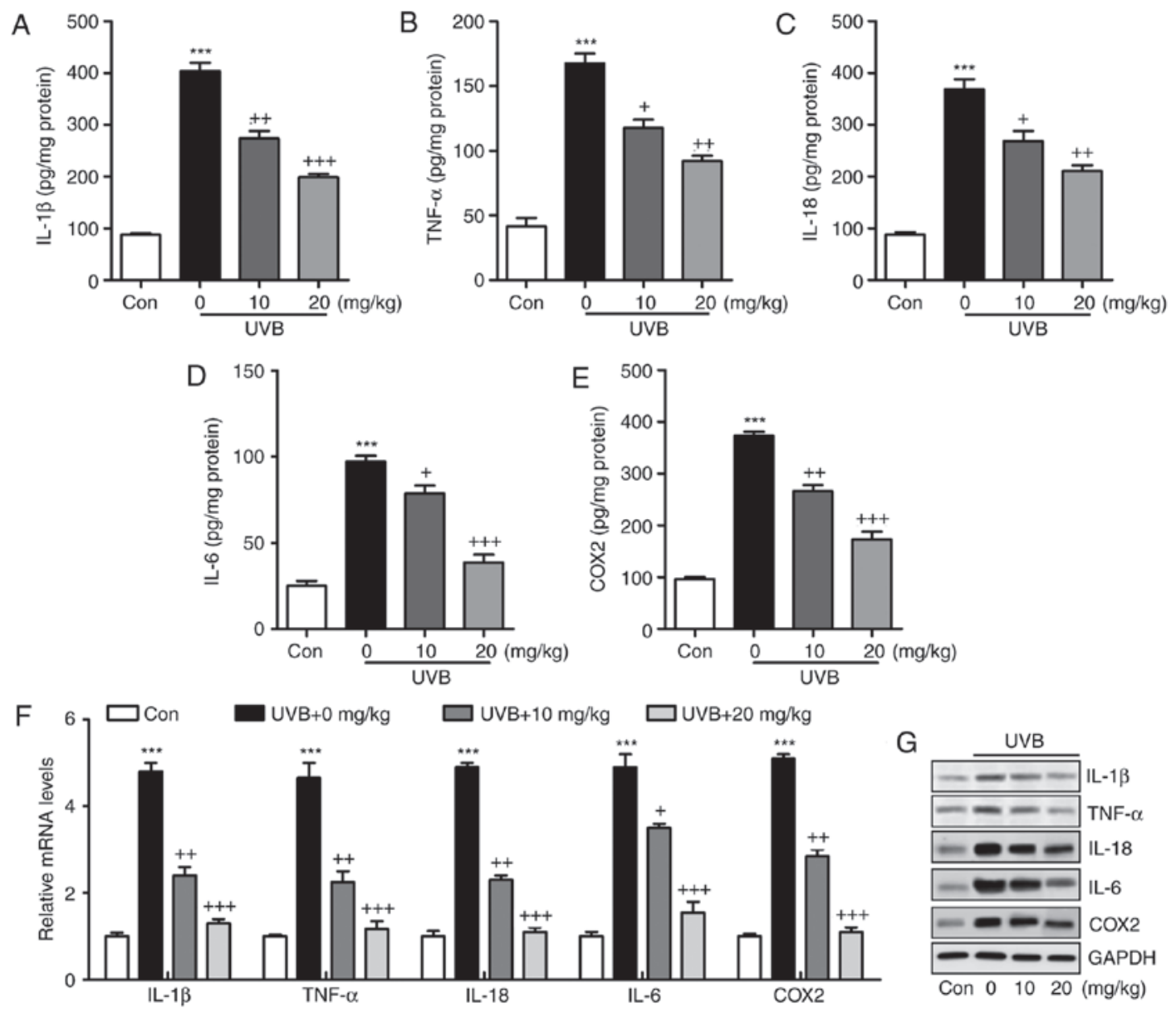

Figure 3. Liquiritin reduces the release of pro-inflammatory cytokines in UVB-induced skin in mice. Expression of pro-inflammatory cytokines (A) IL-1 $\beta$, (B) TNF- $\alpha$, (C) IL-18, (D) IL-6 and (E) COX2 in the skin tissue samples were calculated using ELISA methods. (F) Reverse transcription-quantitative polymerase chain reaction and $(\mathrm{G})$ western blot assays were used to evaluate the gene and protein levels of IL-1 $\beta$, TNF- $\alpha$, IL-18, IL- 6 and COX2 in the skin tissue of mice obtained from mice induced by UVB. Data are presented as the mean \pm standard error of the mean $(\mathrm{n}=10) .{ }^{* * * *} \mathrm{P}<0.001$, compared with the Con group. ${ }^{+} \mathrm{P}<0.05,{ }^{+} \mathrm{P}<0.01$ and ${ }^{++} \mathrm{P}<0.001$, compared with the UVB-only group. UVB, ultraviolet B; Con, control; IL, interleukin; TNF- $\alpha$, tumor necrosis factor- $\alpha$; COX2, cyclooxygenase 2 .

protein levels, determined using western blot analysis. Of note, liquiritin exerted a suppressive effect on the altered release of these signals, with levels comparable to those in the Con group of mice (Fig. 3F and G). These data indicated that liquiritin improved UVB-induced skin injury through reducing the release of pro-inflammatory cytokines.

Activation of the TLR4/MyD88 signaling pathway can lead to the direct activation of NF- $\mathrm{BB}$, contributing to pro-inflammatory cytokine secretion (22). The TLR4/MyD88 and NF- $\mathrm{KB}$ signaling pathways were investigated in the present study. As shown in Fig. 4A, the immunohistochemical analysis indicated that the level of TLR4 was induced in the skin tissue sections of mice exposed to UVB, which was reduced by liquiritin administration. Similar results were observed for TLR4 and MyD88 protein levels using western blot analysis (Fig. 4B). Additionally, the protein levels of $\mathrm{p}-\mathrm{I} \kappa \mathrm{B} \alpha, \mathrm{p}-\mathrm{IKK} \alpha$, and $\mathrm{p}-\mathrm{NF}-\mathrm{\kappa B}$ were high in the UVB-only group. By contrast, I $\kappa \mathrm{B} \alpha$ was reduced under UVB exposure. Liquiritin treatment significantly decreased the phosphorylation of IKK $\alpha$, ІкB $\alpha$, and NF- $\kappa \mathrm{B}$, and increased the levels of I $\kappa \mathrm{B} \alpha$ in a dose-dependent manner (Fig. 4C). Together, these data indicated that the liquiritin-ameliorated inflammation induced by UVB was dependent on TLR4/MyD88/NF- $\mathrm{BB}$ inactivation.
Liquiritin inhibits $U V B$-induced oxidative stress in mice. Oxidative stress is reported to be key in the induction of skin injury induced by UVB $(11,12)$. Therefore, antioxidants and oxidants were measured in the present study. As shown in Fig. 5A and B, it was found that the activity of SOD in serum and skin tissue samples of mice exposed to UVB was lower, compared with that in the Con group of mice. Of note, liquiritin treatment upregulated the activity of SOD in the serum and in the skin tissue samples. By contrast, the levels of MDA were found to be higher following UVB exposure, which were downregulated due to liquiritin administration. The levels of antioxidants $\mathrm{H}_{2} \mathrm{O}_{2}$ and $\mathrm{O}_{2}$ in the skin were found to be accelerated in the UVB-treated mice, and were reduced by liquiritin treatment (Fig. 5C). Oxidative stress was present in the skin of mice according to these results, which was reversed following liquiritin administration. Subsequently, the present study attempted to examine the molecular mechanism by which liquiritin exerted its effect in reducing oxidative stress. As shown in Fig. 6A, RT-qPCR analysis indicated that the gene expression levels of SOD1, SOD2, CAT and nuclear factor erythroid 2-related factor 2 (Nrf2), which are important antioxidants for inhibiting oxidants and suppressing ROS, were decreased following UVB irradiation. In the liquiritin-treated 

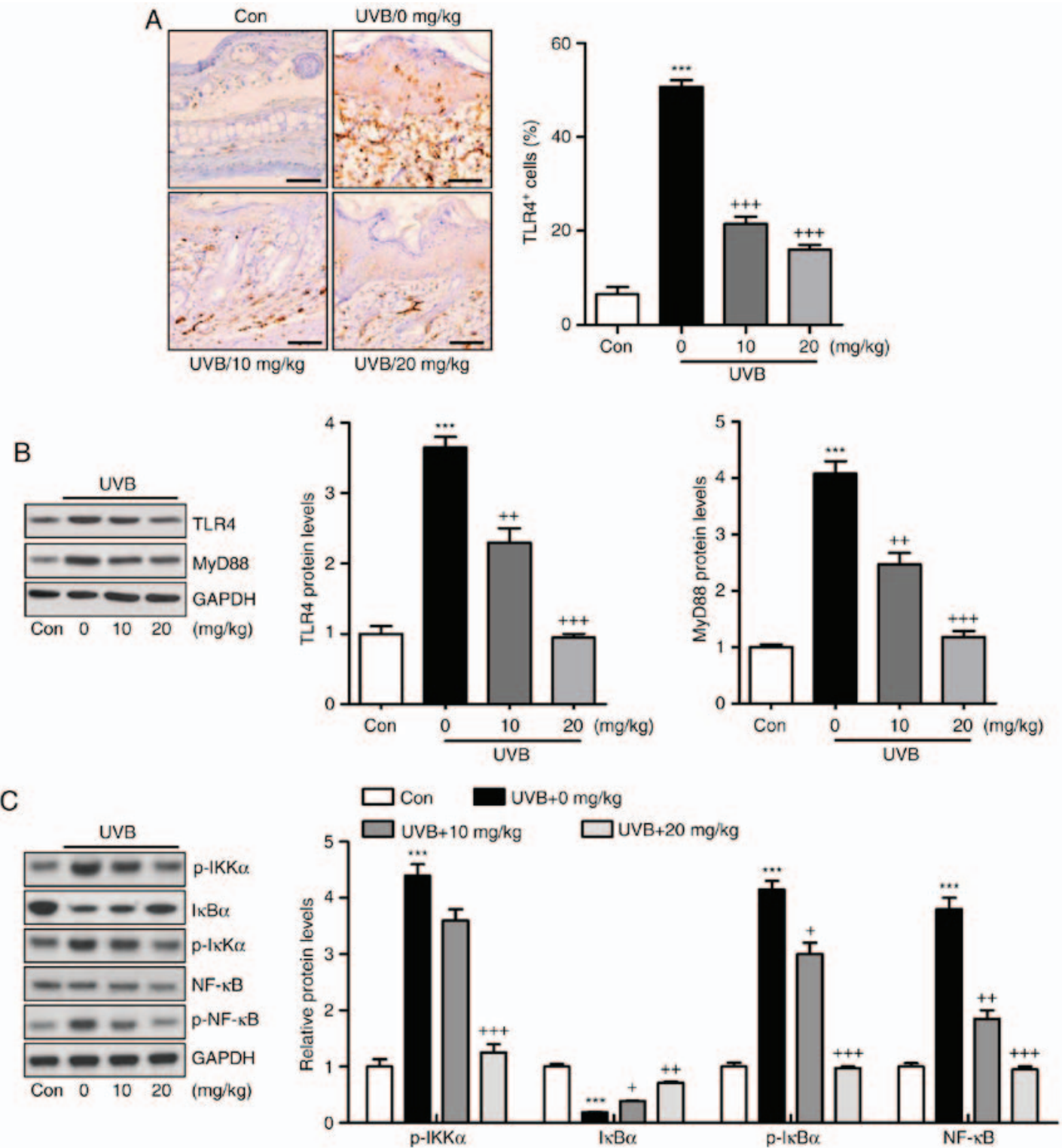

Figure 4. Liquiritin-reduced pro-inflammatory cytokines release is dependent on the TLR4/NF-kB signaling pathway. (A) Immunohistochemical analysis was performed to assess TLR4-positive cells in various groups of mice exposed to UVB irradiation. Scale bar, $100 \mu \mathrm{m}$. (B) Western blot analysis was performed to determine the expression levels of TLR4 and MyD88, shown in the representative images and quantification in the histogram. (C) Protein levels of p-IKK $\alpha$, $\mathrm{I} \kappa \mathrm{B} \alpha, \mathrm{p}-\mathrm{I} \kappa \mathrm{B} \alpha$, and $\mathrm{p}-\mathrm{NF}-\kappa \mathrm{B}$ were assessed using western blot analysis, and quantification of these proteins is shown in the histogram. Data are presented as the mean \pm standard error of the mean $(\mathrm{n}=10) .{ }^{* * *} \mathrm{P}<0.001$, compared with the Con group. ${ }^{+} \mathrm{P}<0.05,{ }^{++} \mathrm{P}<0.01$ and ${ }^{++} \mathrm{P}<0.001$, compared with the UVB-only group.

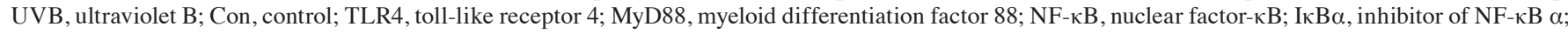
IKK $\alpha$, IкB kinase $\alpha$; p-, phosphorylated.

groups, the expression of these signals was increased, further indicating that liquiritin is potentially involved in ameliorating oxidative stress via enhancing the expression of antioxidants. The molecules XO, iNOS, NOX-4 and NOX-2, which are crucial in promoting ROS generation, were induced under UVB exposure. Liquiritin treatment at various concentrations significantly reduced the expression of these molecules, demonstrating its inhibitory effects on ROS (Fig. 6B). NOX-2 is known to be important in contributing to ROS generation. Therefore, immunohistochemical analysis was performed to further investigate how liquiritin affects the expression of NOX-2 in UVB-induced skin injury (Fig. 6C). The representative images indicated that liquiritin reduced the UVB-induced levels of NOX-2 positive cells, which was in accordance with the results of western blot analysis. Taken together, the results indicated that liquiritin reduced oxidative stress to ameliorate UVB-induced skin injury in vivo.

Liquiritin ameliorates UVB-induced skin injury dependent on MAPK and caspase signaling pathways. Liquiritin has been reported to inhibit cancer progression via the modulation of apoptosis (23). Therefore, it was hypothesized that liquiritin may improve UVB-induced skin injury associated with the apoptotic response. The MAPK signaling pathway is known to affect apoptosis under various conditions (24). In the present study, it was found that MAPKs, p38 and JNK were phosphorylated by UVB in the skin tissue samples from mice, and were reduced by liquiritin treatment (Fig. 7A). The caspase signaling pathway is known to regulate the apoptotic response (25). The results of western blot analysis indicated 

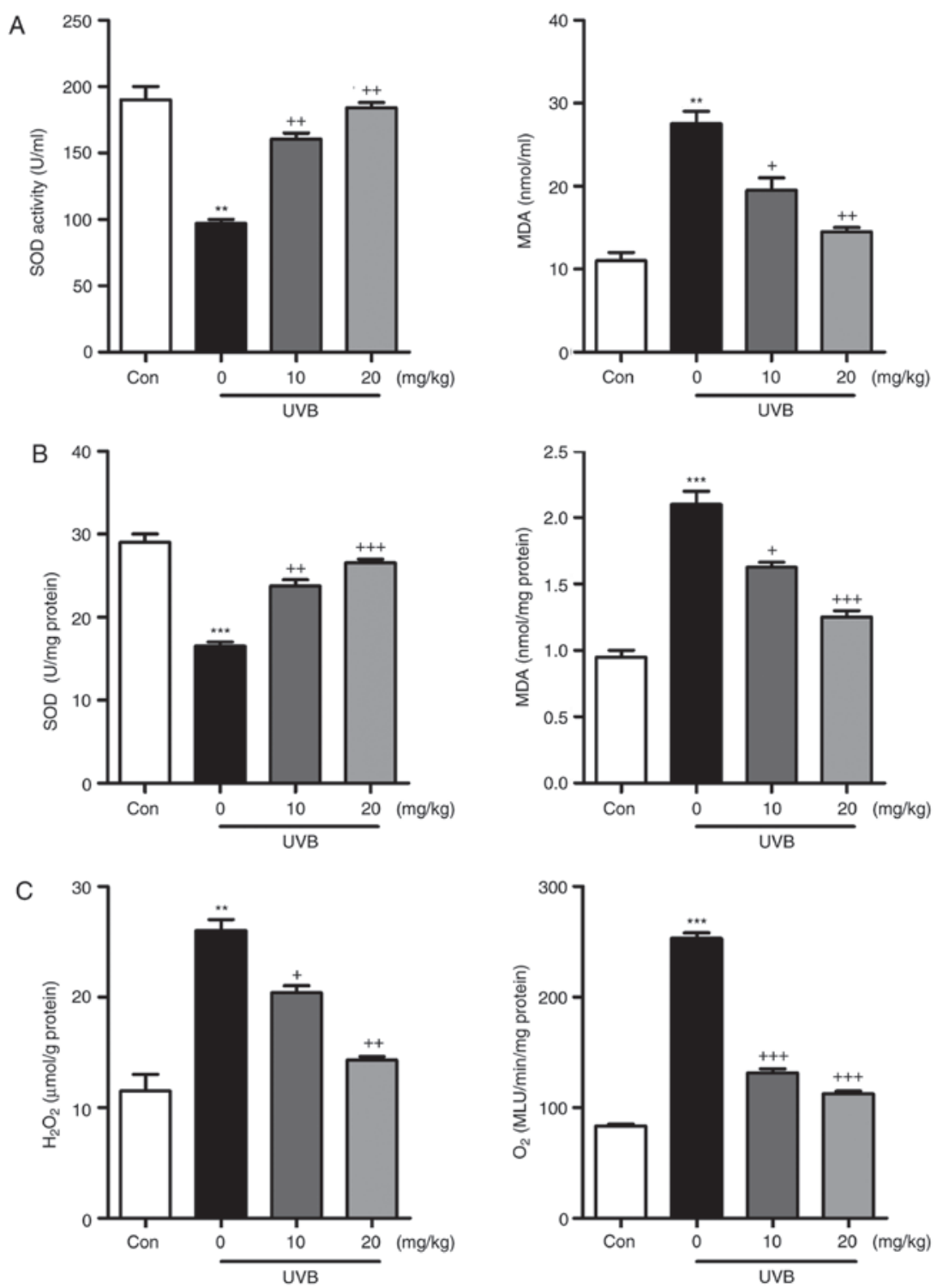

Figure 5. Liquiritin reduces UVB-induced oxidative stress in mice. Activity of SOD and levels of MDA in (A) serum and (B) skin tissue samples were evaluated. (C) Levels of $\mathrm{H}_{2} \mathrm{O}_{2}$ and $\mathrm{O}_{2}$. in the skin were assessed. Data are presented as the mean \pm standard error of the mean $(\mathrm{n}=10)$. ${ }^{* * *} \mathrm{P}<0.01$ and ${ }^{* * * *} \mathrm{P}<0.001$, compared with the Con group. ${ }^{+} \mathrm{P}<0.05,{ }^{++} \mathrm{P}<0.01$ and ${ }^{++} \mathrm{P}<0.001$, compared with the UVB-only group. UVB, ultraviolet $\mathrm{B}$; Con, control; SOD, superoxide dismutase; MDA, malondialdehyde.

that cleaved caspase- 9 , caspase-3 and PARP were expressed at high levels in the UVB-treated mice, suggesting that apoptosis was induced under UVB exposure, leading to eventual cell death. However, liquiritin inhibited the activation of these signals, suppressing apoptosis and cell death (Fig. 7B). Finally, TUNEL analysis directly indicated that liquiritin reduced UVB-induced apoptosis (Fig. 7C). Together, these data indicated that UVB-induced skin injury was closely associated with apoptosis, which was inhibited by liquiritin treatment.

Liquiritin has no significant effect on cell viability and hepatotoxicity. In vivo, liquiritin has been shown to ameliorate UVB-induced skin injury. In the present study, in vitro experiments were performed to further examine the effects of liquiritin on UVB-induced skin damage. First, the cell viability was assessed to investigate the safety or cytotoxicity of liquiritin in cells. As shown in Fig. 8A, the HACAT and L02 cells were exposed to various concentrations of liquiritin for different durations. However, no significant difference was observed among the different groups, with the exception of the highest dose of liquiritin in the L02 cells for the longest duration $(96 \mathrm{~h})$. These data, in part, showed that liquiritin at the concentrations and durations examined in the present study was not cytotoxic to cells. Additionally, no significant difference was found in the levels of ALT and AST in the serum of mice treated with different doses of liquiritin, compared with those in the Con group, further indicating the safety of liquiritin (Fig. 8B).

Liquiritin reduces the inflammatory response in UVB-induced HACAT cells in vitro. The HACAT cells were irradiated with UVB and exposed to liquiritin treatment at different concentrations, as indicated, for analysis. The results of the RT-qPCR analysis suggested that the mRNA levels of IL- $1 \beta$, TNF- $\alpha$, IL-18, IL-6 and COX2 were high in the UVB-irradiated cells, and were downregulated by liquiritin treatment, which was in 

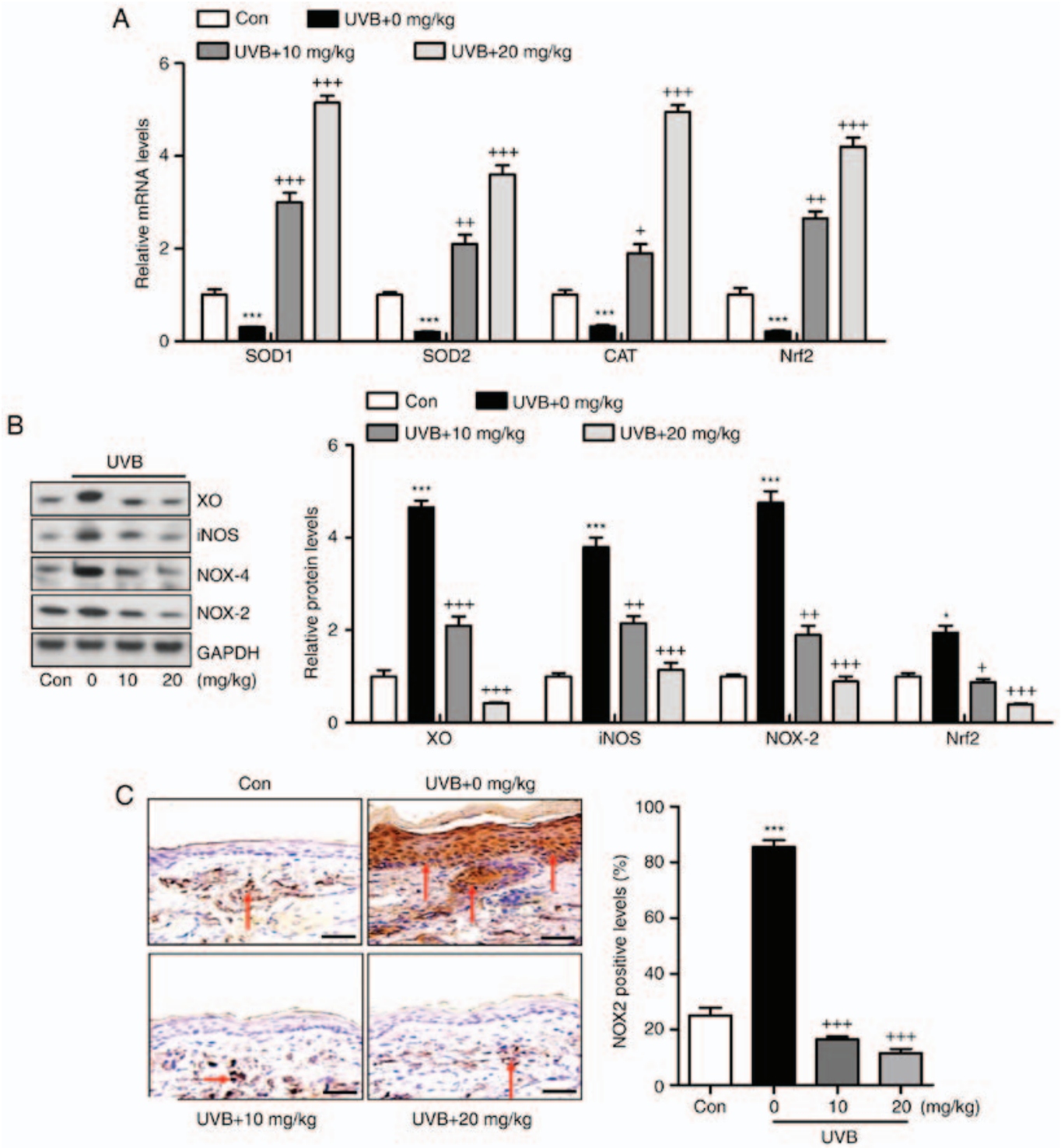

Figure 6. Liquiritin reduces oxidative stress via promoting the expression of antioxidants and suppressing the expression of oxidants in the skin tissue samples. (A) mRNA levels of SOD1, SOD2, CAT and Nrf2 in the skin tissue specimens were calculated using reverse transcription-quantitative polymerase chain reaction analysis. (B) Western blot assays were performed to examine protein levels of XO, iNOS, NOX-2 and NOX-4 in the skin tissue samples of mice treated under various conditions. (C) Expression levels of NOX-2 were measured using immunohistochemical analysis in the skin tissue sections from mice. The red arrows refer to NOX2-positive cells. Scale bar, $100 \mu \mathrm{m}$. Data are presented as the mean \pm standard error of the mean $(\mathrm{n}=10) .{ }^{* * * *} \mathrm{P}<0.001$, compared with the Con group. ${ }^{+} \mathrm{P}<0.05,{ }^{++} \mathrm{P}<0.01$ and ${ }^{++} \mathrm{P}<0.001$, compared with the UVB-only group. UVB, ultraviolet $\mathrm{B}$; Con, control; SOD, superoxide dismutase; CAT, catalase; Nrf2, nuclear factor erythroid 2-related factor 2; XO, xanthine oxidase; iNOS, inducible nitric oxide synthase; NOX, nitric oxide.

accordance with the results of the in vivo experiments (Fig. 9A). In addition, the UVB-induced higher protein levels of TLR4 and MyD88 were reduced by liquiritin treatment in vitro, further confirming that the TLR4/MyD88 signaling pathway was involved in the liquiritin-ameliorated skin injury induced by UVB (Fig. 9B). In addition, the phosphorylated levels of IKK $\alpha$, I $\kappa \mathrm{B} \alpha$, and $\mathrm{NF}-\kappa \mathrm{B}$ proteins were upregulated in the UVB-only group. Similar to the in vivo experiment, I $\mathrm{B} \alpha$ was reduced under UVB exposure. Liquiritin treatment at various concentrations decreased the phosphorylation of IKK $\alpha$, I $\kappa \mathrm{B} \alpha$

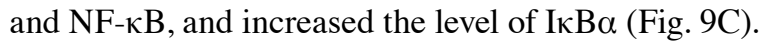

Liquiritin suppresses oxidative stress and apoptosis in HACAT cells exposed to UVB in vitro. The oxidative stress confirmed above was also investigated in vitro. In accordance with the results in vivo, the mRNA levels of SOD1, SOD2,
CAT and Nrf2 induced by UVB were significantly reduced by liquiritin treatment (Fig. 10A). By contrast, the protein levels of antioxidants XO, iNOS, NOX-4 and NOX-2 were induced by UVB exposure, as demonstrated via western blot analysis, and liquiritin administration significantly reduced the expression of these molecules (Fig. 10B). In addition, the protein levels of phosphorylated p38 and JNK induced by UVB in HACAT cells were upregulated, and liquiritin reduced MAPK activation (Fig. 11A). Cleaved caspase-9, caspase-3 and PARP were also expressed at high levels under UVB irradiation. Consistently, liquiritin exhibited a suppressive effect in regulating caspase-9, caspase-3 and PARP cleavage in vitro (Fig. 11B). Finally, it was found that the confluence of HACAT cells exposed to UVB, determined using crystal violet staining, was decreased, which was reversed by liquiritin in a dose-dependent manner (Fig. 11C). These data indicated that 
A

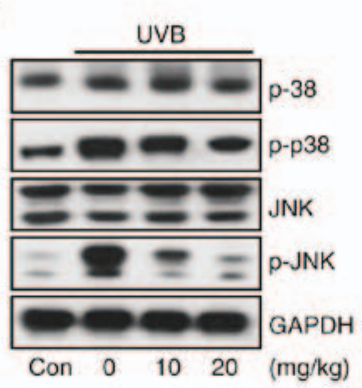

B
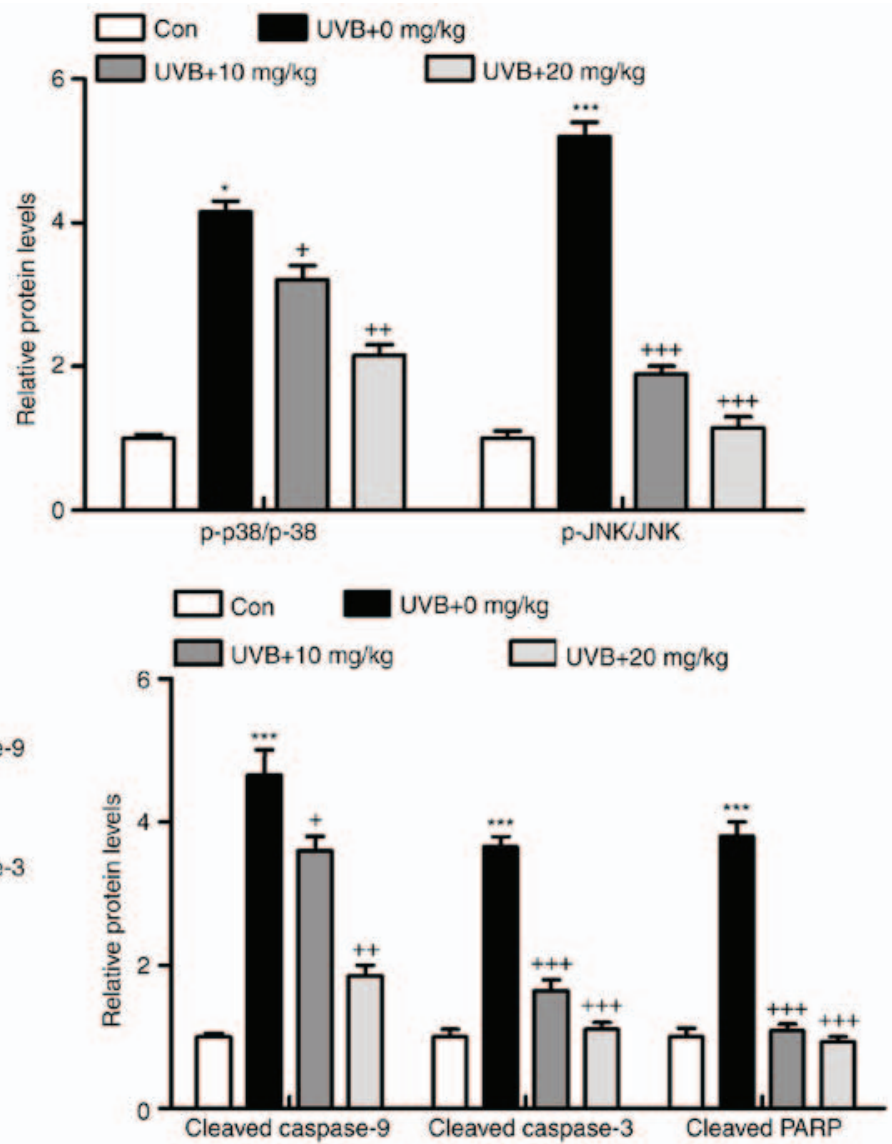

C

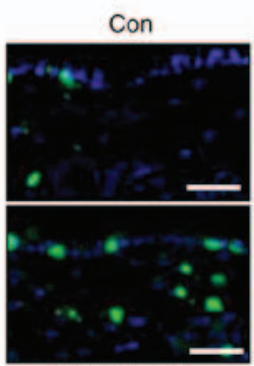

UVB/10 mg/kg
UVB/0 mg/kg

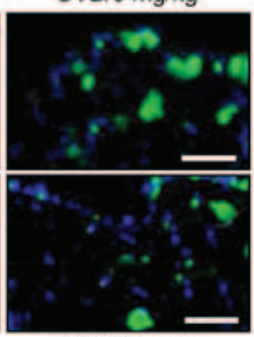

UVB/20 mg/kg

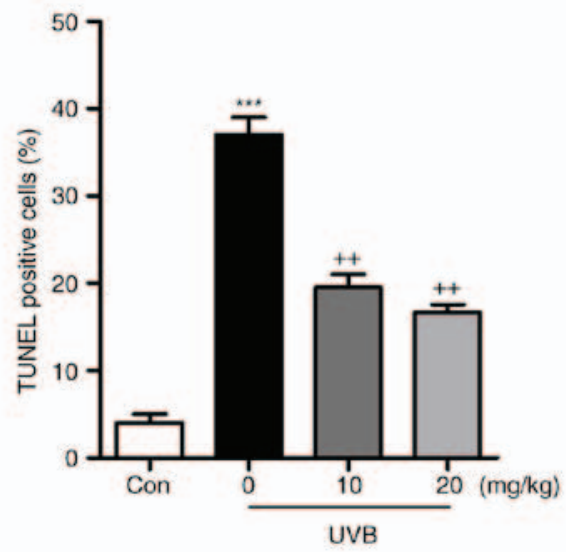

Figure 7. Liquiritin ameliorates UVB-induced skin injury dependent on mitogen-activated protein kinase and caspase signaling pathways. (A) Expression levels of p-p38 and p-JNK were assessed using western blot analysis. (B) Immumoblotting analysis was performed to evaluate the levels of cleaved caspase-9, caspase-3 and PARP in the skin tissue samples isolated from mice exposed to UVB irradiation treated with or without liquiritin. (C) TUNEL-positive levels were evaluated in the skin tissue sections of mice to examine the effect of liquiritin on apoptosis induced by UVB. Scale bar, $100 \mu \mathrm{m}$. Data are presented as the mean \pm standard error of the mean $(\mathrm{n}=10) .{ }^{*} \mathrm{P}<0.05$ and ${ }^{* * * *} \mathrm{P}<0.001$, compared with the the Con group. ${ }^{+} \mathrm{P}<0.05,{ }^{++} \mathrm{P}<0.01$ and ${ }^{+++} \mathrm{P}<0.001$, compared with the UVB-only group. UVB, ultraviolet B; Con, control, JNK, c-Jun N-terminal kinase; PARP, poly(ADP-ribose) polymerase; p-, phosphorylated; TUNEL, Terminal deoxynucleotidyl transferase dUTP nick end labeling.

liquiritin reduced UVB-induced oxidative stress and apoptosis in vitro, which was consistent with the findings of the in vivo experiments.

\section{Discussion}

Skin cancer is known to be one of the most common types of cancer in the world. Solar UVB irradiation is a ubiquitous environmental carcinogen, leading to various cutaneous disorders, including melanoma and non-melanomatous skin cancer (26). According to previous studies, plant-derived products have potential antioxidant, anticancer, antimutagenic and anti-inflammatory properties, which have been examined for the prevention of skin injury induced by UVB (27). In the present study, liquiritin, abundant in crude Glycyrrhiza Radix, may be a potential candidate for use in suppressing UVB-induced hairless mice to examine its effects on the release of pro-inflammatory cytokines, development of oxidative stress and progression of apoptosis through various signaling pathways. The findings of present study indicated that the 
A
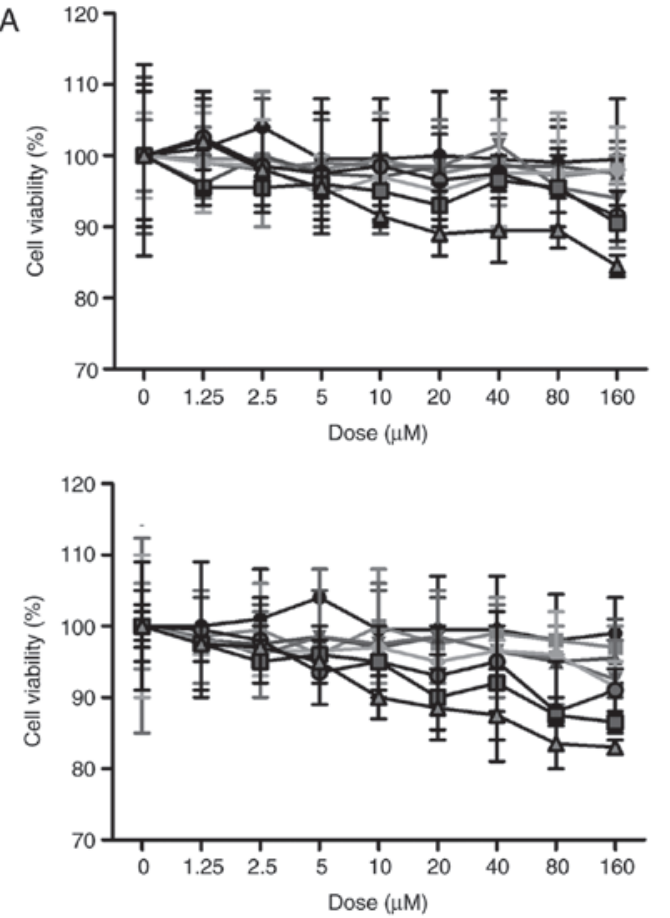
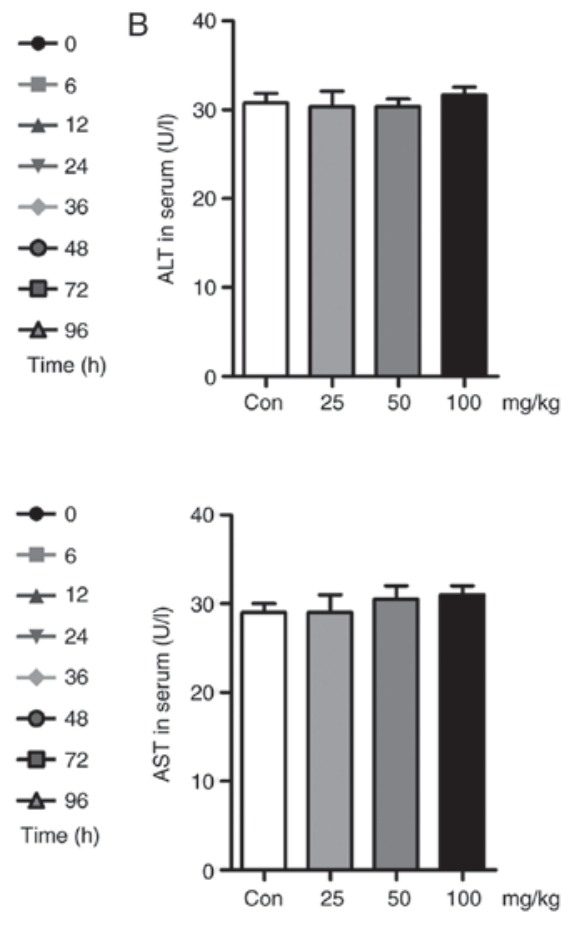

Figure 8. Liquiritin has no significant effect on cell viability or hepatotoxicity. (A) HACAT (above) and L02 (below) cells were exposed to liquiritin at different concentrations for various durations, as indicated. MTT analysis was then used to evaluate cell viability. (B) Levels of ALT and AST in the serum of mice treated with different concentrations of liquiritin-only were measured to calculate the hepatotoxicity of liquiritin in mice. Data are presented as the mean \pm standard error of the mean $(\mathrm{n}=8-10)$. ${ }^{*} \mathrm{P}<0.05$, compared with the Con group. UVB, ultraviolet B; Con, control; ALT, alanine aminotransferase; AST, aspartate aminotransferase.

administration of liquiritin inhibited the UVB-exposed inflammatory response, oxidative stress and apoptotic response, which were dependent on inhibition of the TLR4/NF- $\kappa \mathrm{B}, \mathrm{ROS}-$ related and MAPK/caspase signaling pathways.

Accumulating evidence indicates that on exposure to UVB irradiation, the inflammatory response leads to skin damage by generating the secretion of pro-inflammatory cytokines, including IL-1 $\beta$, TNF- $\alpha$, IL-18, IL-6 and COX2. The increased expression of pro-inflammatory cytokines can accelerate inflammatory cell infiltration, contributing to tissue damage or organ injury, including airway inflammation of the lung $(28,29)$. In addition, in skin damage induced under various situations, the hyper-proliferation of keratinocytes is induced, which is closely associated with the secretion of pro-inflammatory cytokines (30). Similarly, in the present study, UVB irradiation significantly promoted the release of pro-inflammatory cytokines, which were effectively reduced by liquiritin treatment in the skin tissues of UVB-induced mice in vivo. In vitro, the UVB-exposed HACAT cells exhibited higher levels of pro-inflammatory cytokines. Liquiritin treatment suppressed the expression of these cytokines. The activation of NF- $\kappa \mathrm{B}$ leads to increased synthesis and secretion of pro-inflammatory cytokines (31). Upon stimulation of TLR-4, MyD88 binds to the cytoplasmatic domain of TLR-4, which activates IKK. The activated IKK kinase leads to the phosphorylation and degradation of $\mathrm{I} \kappa \mathrm{B}$ in the proteasome, and to the release of NF- $\kappa \mathrm{B}$ from the NF-IKB- $\kappa \mathrm{B}$ complex, enabling the translocation of NF- $\kappa \mathrm{B}$ to the nucleus, where the expression of genes encoding pro-inflammatory cytokines is induced $(28,31)$. In the present study, the TLR4/MyD88 signaling pathway showed a high level of activation following
UVB-irradiation in vivo and in vitro. Subsequently, the $\mathrm{NF}-\kappa \mathrm{B}$ signaling pathway was phosphorylated and released from the NF-I $\kappa \mathrm{B}-\kappa \mathrm{B}$ complex, which enhanced pro-inflammatory cytokine secretion and skin injury.

ROS, including $\mathrm{H}_{2} \mathrm{O}_{2}$ and superoxide anions $\left(\mathrm{O}_{2}{ }^{--}\right)$ produced by cells are involved in the regulation of different cellular functions, including apoptosis, proliferation, transcription activation and intracellular signaling (32). Functioning as a barrier, the skin protects us from being injured by environmental insults, including UV light and toxic chemicals, which induces the generation of ROS (33). ROS-induced damage is reported to be involved in the progression and formation of skin tumors, and in the pathogenesis of inflammatory skin diseases, including atopic dermatitis, contact dermatitis and psoriasis $(34,35)$. Aerobic organisms have effective antioxidant networks to defend against oxidative stress, involving primary enzymes, including SOD and CAT, and inducible phase II detoxifying enzymes, including HO-1 and the activation of Nrf-2 (36). For example, SOD has an antioxidant role in organisms; SOD is necessary as superoxide reacts with sensitive and important cellular targets, including $\mathrm{NO}$ radicals. Additionally, $\mathrm{H}_{2} \mathrm{O}_{2}$ can be detoxified to $\mathrm{H}_{2} \mathrm{O}$ by the scavenging enzyme of CAT. These enzymes act together in the metabolic pathway of free radicals. HO-1 is regulated by the activation of its transcription factor Nrf2 to inhibit ROS generation (37). By contrast, MDA is produced by ROS degrading polyunsaturated lipids and is used as a biomarker to measure the level of oxidative stress in an organism (34). In the present study, $\mathrm{H}_{2} \mathrm{O}_{2}, \mathrm{O}_{2}{ }^{--}$and MDA were found to be higher in the UVB-induced mice, and reduced by liquiritin administration, indicating the effects of 

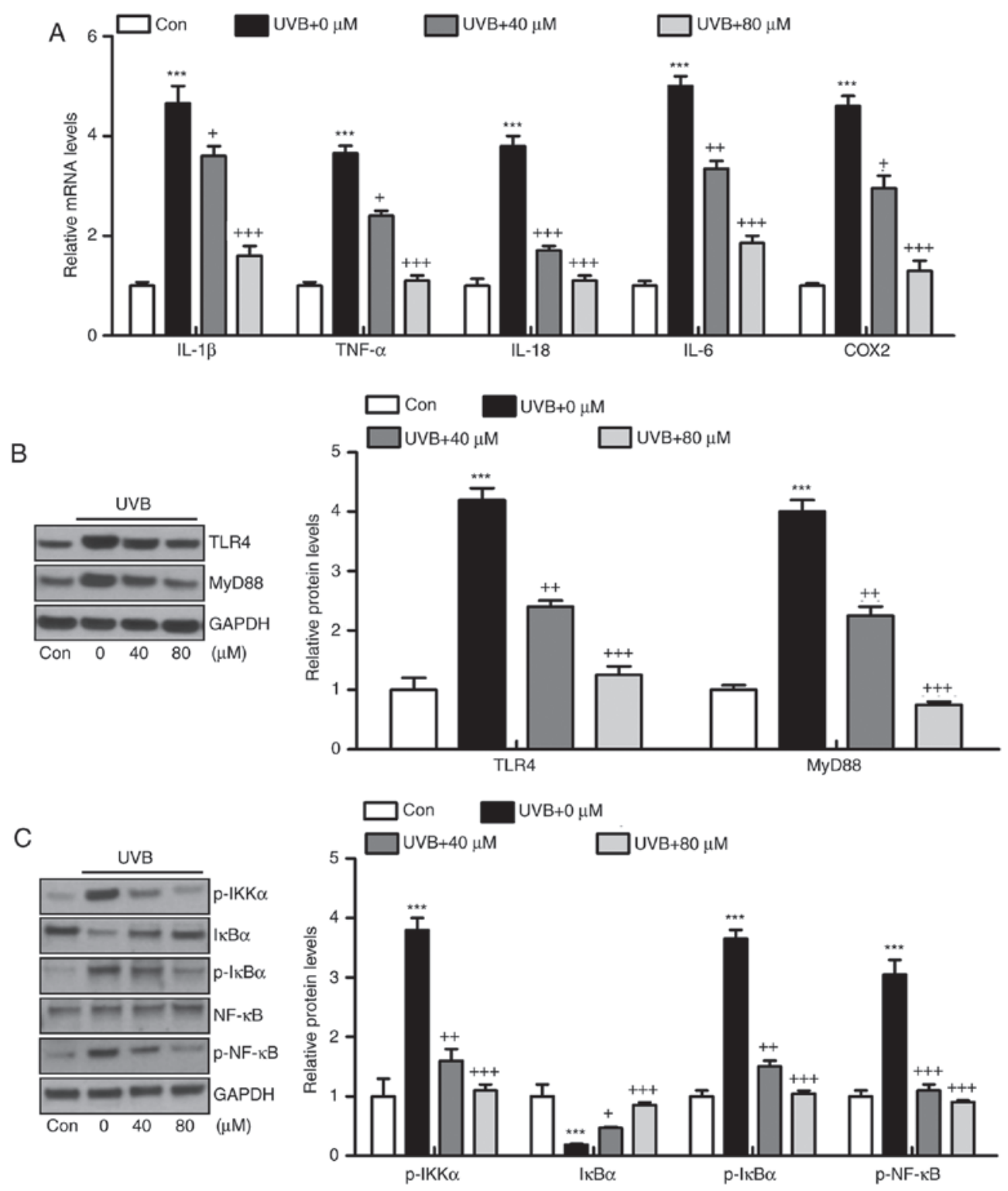

Figure 9. Liquiritin reduces inflammatory response in UVB-induced HACAT cells in vitro. HACAT cells were pre-treated with liquiritin at 40 and $80 \mu \mathrm{M}$ concentrations for $2 \mathrm{~h}$, followed by UVB irradiation together for another $22 \mathrm{~h}$. All cells were then harvested and further analysis was performed to evaluate the role of liquiritin in regulating UVB-induced cells in vitro. (A) Pro-inflammatory cytokines IL-1 $\beta$, TNF- $\alpha$, IL-18, IL- 6 and COX2 were measured using reverse transcription-quantitative polymerase chain reaction analysis. (B) Western blot analysis was performed to evaluate protein levels of TLR4 and MyD88 in vitro. (C) Levels of $\mathrm{p}-\mathrm{IKK} \alpha, \mathrm{I \kappa} \mathrm{B} \alpha, \mathrm{p}-\mathrm{I} \mathrm{KB} \alpha$ and $\mathrm{p}-\mathrm{NF}-\kappa \mathrm{B}$ were assessed using western blot analysis. Data are presented as the mean \pm standard error of the mean $(\mathrm{n}=8) .{ }^{* * * *} \mathrm{P}<0.001$, compared with the Con group. ${ }^{+} \mathrm{P}<0.05,{ }^{++} \mathrm{P}<0.01$ and ${ }^{+++} \mathrm{P}<0.001$, compared with the UVB-only group. UVB, ultraviolet $\mathrm{B}$; Con, control; IL, interleukin; TNF- $\alpha$ tumor necrosis factor- $\alpha$; COX2, cyclooxygenase 2; TLR4, toll-like receptor 4; MyD88, myeloid differentiation factor 88; NF- $\kappa B$, nuclear

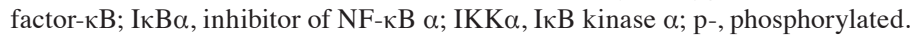

liquiritin to diminish ROS. On the other, RT-qPCR analysis indicated that UVB irradiation reduced the expression of these enzymes at the gene level, and these levels were significantly enhanced by liquiritin administration, reducing ROS generation to ameliorate oxidative stress. $\mathrm{XO}$ is an important enzyme, which catalyzes the oxidation of hypoxanthine to xanthine and further catalyzes the oxidation of xanthine to uric acid, producing $\mathrm{H}_{2} \mathrm{O}_{2}$ and superoxide (38). The enzyme is vital in the development of various diseases $(38,39)$. NO has been implicated as important mediator in the inflammatory process through accelerating the production of ROS. The excessive production of NO is produced by iNOS. NOX-dependent signaling is critical in the development of tissue injury, including that in the heart, liver and lung (40).
NOX-2 and NOX-4, as the major NADPH oxidase isoform expressed in the tissue samples, are principal sources of oxidative stress $(41,42)$. In the present study, it was found that UVB irradiation significantly upregulated XO, iNOS, NOX2 and NOX4, enhancing the progression of ROS. Liquiritin exhibited an inhibitory effect on the expression of these signals. Subsequently, oxidative stress was improved.

The MAPK signaling pathway is also key in a number of biological processes, followed by caspase cleavage, contributing to PARP apoptotic signals $(24,25,43)$. P38 and JNK MAPKs have been reported to mediate cellular apoptosis, proliferation and differentiation (44). Apoptosis, or programmed cell death, is a regulated process, which allows a cell to self-degrade to enable the body to eliminate unwanted or dysfunctional 
A

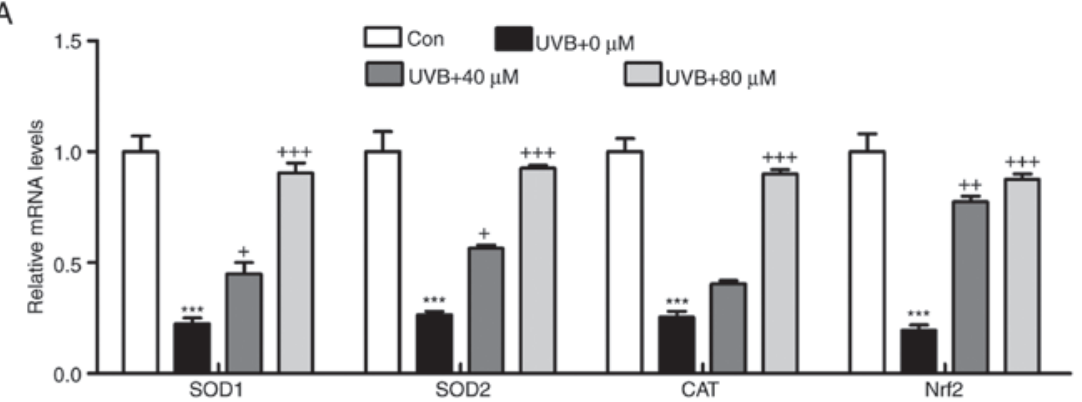

B

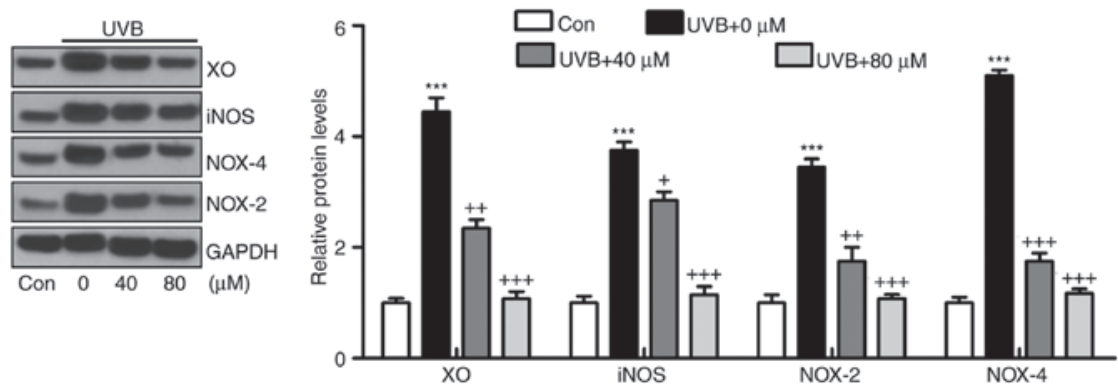

Figure 10. Liquiritin suppresses oxidative stress via promotion of antioxidants and reduction of oxidants in vitro. (A) mRNA levels of SOD1, SOD2, CAT and Nrf2 were measured using reverse transcription-quantitative polymerase chain reaction analysis. (B) Protein levels of XO, iNOS, NOX-4 and NOX-2 were evaluated using using western blot analysis. Data are presented as the mean \pm standard error of the mean $(\mathrm{n}=8)$. ${ }^{* * *} \mathrm{P}<0.001$, compared with the Con group ${ }^{+} \mathrm{P}<0.05,{ }^{++} \mathrm{P}<0.01$ and ${ }^{+++} \mathrm{P}<0.001$, compared with the UVB-only group. UVB, ultraviolet B; Con, control; SOD, superoxide dismutase; CAT, catalase; Nrf2, nuclear factor erythroid 2-related factor 2; XO, xanthine oxidase; iNOS, inducible nitric oxide synthase; NOX, nitric oxide.

A

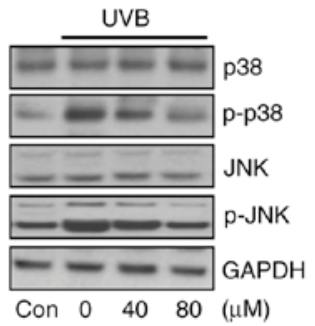

B

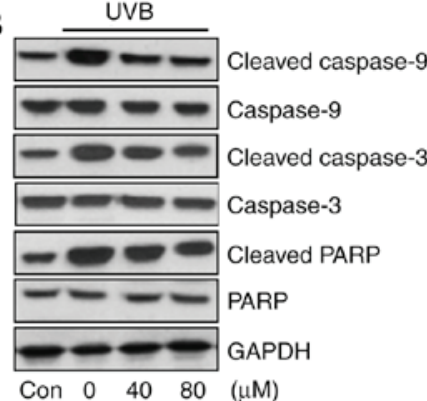

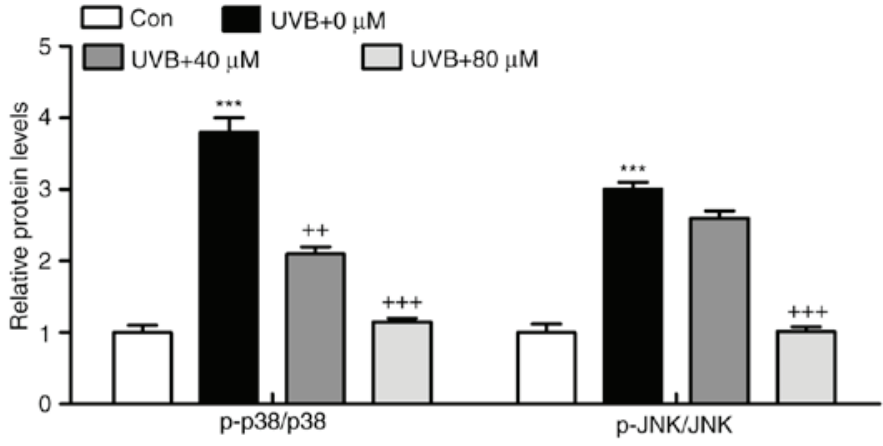

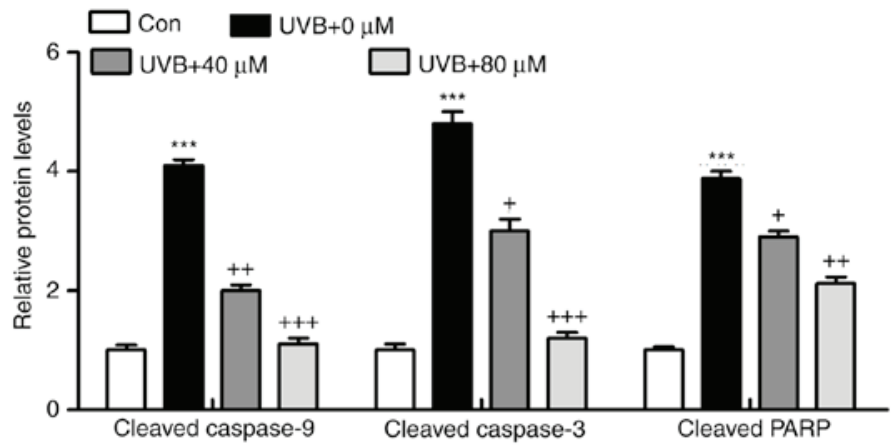

$\mathrm{C}$

UVB

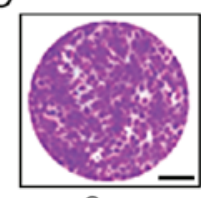

Con
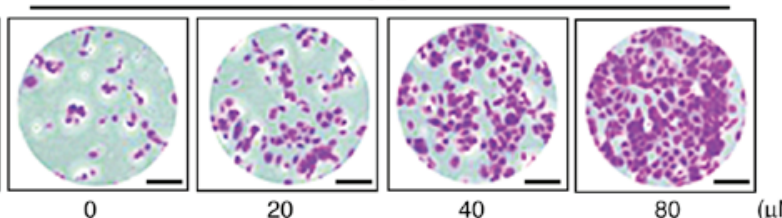

$(\mu \mathrm{M})$

Figure 11. Liquiritin improves UVB-induced HACAT injury through apoptosis suppression. (A) Western blot analysis was performed to evaluate the levels of p-p38 and p-JNK in the cells under various conditions. (B) Cleaved caspase-9, caspase-3 and PARP were measured using western blot analysis in vitro. (C) Confluence of HACAT treated under different conditions. Data are presented as the mean \pm standard error of the mean $(\mathrm{n}=10)$. ${ }^{* * *} \mathrm{P}<0.001$, compared with the Con group. ${ }^{+} \mathrm{P}<0.05,{ }^{++} \mathrm{P}<0.01$ and ${ }^{+++} \mathrm{P}<0.001$, compared with the UVB-only group. UVB, ultraviolet $\mathrm{B}$; Con, control; JNK, c-Jun N-terminal kinase; PARP, poly(ADP-ribose) polymerase; p-, phosphorylated. 
cells (45). The intrinsic pathway is initiated through the release of signaling factors by mitochondria within the cell, and its two apoptotic pathways are executed predominantly by a class of cysteine proteases, termed caspases (46). It is well known that activation of the p38 MAPK and JNK pathways leads to the induction of apoptosis through the phosphorylation of a variety of pro-apoptotic downstream effectors, whereas the ERK1/2 signal pathway is more often associated with cell survival (47). In the present study, UVB induction promoted the phosphorylation of p38 and JNK. Mitochondrial dysfunction induces the activation of caspase- 9 and, subsequently, activates effector caspases, including caspase-3. Following caspase-3 activation, the cleavage of PARP occurs, contributing to apoptosis (48). In accordance with the description above, the present study showed that UVB exposure elevated the cleavage of caspase-9, caspase-3 and PARP, leading to apoptosis, evidenced by TUNEL analysis. Liquiritin was found to suppress the activation of p38 and JNK. Consequently, caspase cleavage and PARP cleavage were reduced. Eventually, UVB-induced cell death may be prevented.

In conclusion, the findings of the present study indicated that liquiritin application to mice exposed to UVB leads to a significant reduction in the release of pro-inflammatory cytokines through the inactivation of TLR4/MyD88/NF- $\mathrm{B}$. Additionally, oxidative stress was suppressed by liquiritin through promoting the expression of antioxidants and inhibiting levels of oxidants. Finally, cell survival was enhanced due to liquiritin treatment via the suppression of apoptosis. Overall, the findings suggested that liquiritin may be developed as an effective photochemopreventive candidate to prevent UVB-induced skin damage.

\section{Acknowledgements}

The authors thank Dr. Ruixia Yang for her technical support.

\section{Funding}

No funding was received.

\section{Availability of data and materials}

The datasets used during the present study are available from the corresponding author upon reasonable request.

\section{Authors' contributions}

XQL, LMC and MJ conceived and designed the study. XQL, LMC, JL, YLM, YHK and HL performed the experiments. XQL and LMC wrote the study. XQL, LMC, YHK and MJ reviewed and edited the manuscript. All authors read and approved the manuscript and agree to be accountable for all aspects of the research in ensuring that the accuracy or integrity of any part of the work are appropriately investigated and resolved.

\section{Ethics approval and consent to participate}

All animal experiments were performed following the Guide for the Care and Use of Laboratory Animals, issued by the National Institutes of Health in 1996 and approved by Huai'an
First People's Hospital, Nanjing Medical University (Nanjing, China). The care and handling of mice were in accordance with the ethical guidelines of Huai'an First People's Hospital.

\section{Patient consent for publication}

Not applicable.

\section{Competing interests}

The authors declare that there are no competing interests.

\section{References}

1. Yager J: The skin as an immune organ. Advances in Veterinary Dermatology. Ihrke PJ, Mason IS and White SD (eds.): Pergamon Press; Oxford: pp311993.

2. Afaq F, Adhami VM and Mukhtar H: Photochemoprevention of ultraviolet B signaling and photocarcinogenesis. Mutat Res 571: 153-173, 2005.

3. Muthusamy V and Piva TJ: A comparative study of UV-induced cell signalling pathways in human keratinocyte-derived cell lines. Arch Dermatol Res 305: 817-833, 2013.

4. Muthusamy V and Piva TJ: The UV response of the skin: A review of the MAPK, NFkappaB, and TNFalpha signal transduction pathways. Arch Dermatol Res 302: 5-17, 2010.

5. Masferrer JL, Leahy KM, Koki AT, Zweifel BS, Settle SL, Woerner BM, Edwards DA, Flickinger AG, Moore RJ and Seibert K: Antiangiogenic and antitumor activities of cyclooxygenase-2 inhibitors. Cancer Res 60: 1306-1311, 2000.

6. Kim SB, Kang OH, Joung DK, Mun SH, Seo YS, Cha MR, Ryu SY, Shin DW and Kwon DY: Anti-inflammatory effects of tectroside on UVB-induced HaCaT cells. Int J Mol Med 31: 1471-1476, 2013.

7. Buckman SY, Gresham A, Hale P, Hruza G, Anast J, Masferrer J and Pentland AP: COX-2 expression is induced by UVB exposure in human skin: Implications for the development of skin cancer. Carcinogenesis 19: 723-729, 1998.

8. Karpurapu M, Wang X, Deng J, Park H, Xiao L, Sadikot RT, Frey RS, Maus UA, Park GY, Scott EW and Christman JW: Functional PU.1 in macrophages has a pivotal role in NF- $\kappa$ B activation and neutrophilic lung inflammation during endotoxemia. Blood 118: 5255-5266, 2011.

9. Medvedev AE, Lentschat A, Wahl LM, Golenbock DT and Vogel SN: Dysregulation of LPS-induced Toll-like receptor 4-MyD88 complex formation and IL-1 receptor-associated kinase 1 activation in endotoxin-tolerant cells. J Immunol 169: 5209-5216, 2002.

10. Lee J, Giordano S and Zhang J: Autophagy, mitochondria and oxidative stress: Cross-talk and redox signalling. Biochem J 441: 523-540, 2012.

11. Acker T, Fandrey $\mathrm{J}$ and Acker $\mathrm{H}$ : The good, the bad and the ugly in oxygen-sensing: ROS, cytochromes and prolyl-hydroxylases. Cardiovasc Res 71: 195-207, 2006.

12. Burotto M, Chiou VL, Lee JM and Kohn EC: The MAPK pathway across different malignancies: A new perspective. Cancer 120: 3446-3456, 2014.

13. Hsieh SC, Huang MH, Cheng CW, Hung JH, Yang SF and Hsieh YH: $\alpha$-Mangostin induces mitochondrial dependent apoptosis in human hepatoma SK-Hep-1 cells through inhibition of p38 MAPK pathway. Apoptosis 18: 1548-1560, 2013.

14. Lamy E, Herz C, Lutz-Bonengel S, Hertrampf A, Márton MR and Mersch-Sundermann V: The MAPK pathway signals telomerase modulation in response to isothiocyanate-induced DNA damage of human liver cancer cells. PLoS One 8: e53240, 2013.

15. Hui K, Yang Y, Shi K, Luo H, Duan J, An J, Wu P, Ci Y, Shi L and Xu C: The p38 MAPK-regulated PKD1/CREB/Bcl-2 pathway contributes to selenite-induced colorectal cancer cell apoptosis in vitro and in vivo. Cancer Lett 354: 189-199, 2014.

16. Wang W, Hu X, Zhao Z, Liu P, Hu Y, Zhou J, Zhou D, Wang Z, Guo D and Guo H: Antidepressant-like effects of liquiritin and isoliquiritin from Glycyrrhiza uralensis in the forced swimming test and tail suspension test in mice. Prog Neuropsychopharmacol Biol Psychiatry 32: 1179-1184, 2008. 
17. Sun YX, Tang Y, Wu AL, Liu T, Dai XL, Zheng QS and Wang ZB: Neuroprotective effect of liquiritin against focal cerebral ischemia/reperfusion in mice via its antioxidant and antiapoptosis properties. J Asian Nat Prod Res 12: 1051-1060, 2010.

18. Wu TY, Khor TO, Saw CL, Loh SC, Chen AI, Lim SS, Park JH, Cai L and Kong AN: Anti-inflammatory/anti-oxidative stress activities and differential regulation of Nrf2-mediated genes by non-polar fractions of tea Chrysanthemum zawadskii and licorice Glycyrrhiza uralensis. AAPS J 13: 1-13, 2011.

19. Dong SJ, Inoue A, Zhu Y, Tanji M and Kiyama R: Activation of rapid signally pathways and the subsequent transcriptional regulation for the proliferation of breast cancer MCF-7 cells by the treatment with an extract of Glycyrrhiza glabra root. Food Chem Toxicol 45: 2470-2478, 2007.

20. Livak KJ and Schmittgen TD: Analysis of relative gene expression data using real-time quantitative PCR and the 2(-Delta Delta C(T)) method. Methods 25: 402-408, 2001.

21. Weiler C, Nerlich AG, Bachmeier BE and Boos N: Expression and distribution of tumor necrosis factor alpha in human lumbar intervertebral discs: A study in surgical specimen and autopsy controls. Spine (Phila Pa 1976) 30: 44-54, 2005.

22. Le Maitre CL, Hoyland JA and Freemont AJ: Catabolic cytokine expression in degenerate and herniated human intervertebral discs: IL-1beta and TNFalpha expression profile. Arthritis Res Ther 9: R77, 2007.

23. Fu Y, Hsieh ZC, Guo JQ, Kunicki J, Lee YWT, Darzynkiewicz Z and $\mathrm{Wu} \mathrm{JM}$ : Licochalcone-A, a novel flavonoid isolated from licorice root (Glycyrrhiza glabra), causes G2 and late-G1 arrests in androgen-independent PC-3 prostat cancer cells. Biochem Biophys Res Commun 322: 263-270, 2004.

24. Kuo WH, Chen JH, Lin HH, Chen BC, Hsu JD and Wang CJ: Induction of apoptosis in the lung tissue from rats exposed to cigarette smoke involves p38/JNK MAPK pathway. Chem Biol Interact 155: 31-42, 2005 .

25. American Cancer Society: Cancer Facts and Figures, 2010. American Cancer Society, Atlanta, GA, 2010.

26. Suzuki Y, Nakabayashi Y, Nakata K, Reed JC and Takahashi R $\mathrm{X}$-linked inhibitor of apoptosis protein (XIAP) inhibits caspase-3 and -7 in distinct modes. J Biol Chem 276: 27058-27063, 2001.

27. Perrone D, Ardito F, Giannatempo G, Dioguardi M, Troiano G, Lo Russo L, DE Lillo A, Laino L and Lo Muzio L: Biological and therapeutic activities, and anticancer properties of curcumin (Review). Exp Ther Med 10: 1615-1623, 2015.

28. Nilsson MB, Langley RR and Fidler IJ: Interleukin-6, secreted by human ovarian carcinoma cells, is a potent proangiogenic cytokine. Cancer Res 65: 10794-10800, 2005.

29. Cho JW, Lee KS and Kim CW: Curcumin attenuates the expression of IL-1beta, IL-6, and TNF-alpha as well as cyclin E in TNF-alpha-treated HaCaT cells; NF-kappaB and MAPKs as potential upstream targets. Int J Mol Med 19: 469-474, 2007.

30. Chen W, Tang Q, Gonzales MS and Bowden GT: Role of p38 MAP kinases and ERK in mediating ultraviolet-B induced cyclooxygenase-2 gene expression in human keratinocytes. Oncogene 20: 3921-3926, 2001.

31. Wullaert A, Bonnet MC and Pasparakis M: NF- $\kappa$ B in the regulation of epithelial homeostasis and inflammation. Cell Res 21 146-158, 2011

32. Rajagopalan S, Kurz S, Münzel T, Tarpey M, Freeman BA, Griendling KK and Harrison DG: Angiotensin II-mediated hypertension in the rat increases vascular superoxide production via membrane NADH/NADPH oxidase activation. Contribution to alterations of vasomotor tone. J Clin Invest 97: 1916-1923, 1996.
33. Touyz RM and Briones AM: Reactive oxygen species and vascular biology: Implications in human hypertension. Hypertens Res 34: 5-14, 2011.

34. Jia SJ, Jiang DJ, Hu CP, Zhang XH, Deng HW and Li YJ: Lysophosphatidylcholine-induced elevation of asymmetric dimethylarginine level by the NADPH oxidase pathway in endothelial cells. Vascul Pharmacol 44: 143-148, 2006.

35. Hanna IR, Taniyama Y, Szöcs K, Rocic P and Griendling KK: $\mathrm{NAD}(\mathrm{P}) \mathrm{H}$ oxidase-derived reactive oxygen species as mediators of angiotensin II signaling. Antioxid Redox Signal 4: 899-914, 2002.

36. Luo Z, Teerlink T, Griendling K, Aslam S, Welch WJ and Wilcox CS: Angiotensin II and NADPH oxidase increase ADMA in vascular smooth muscle cells. Hypertension 56: 498-504, 2010

37. Was H, Dulak $J$ and Jozkowicz A: Heme oxygenase-1 in tumor biology and therapy. Curr Drug Targets 11: 1551-1570, 2010.

38. Wang G, Qian P, Jackson FR, Qian G and Wu G: Sequential activation of JAKs, STATs and xanthine dehydrogenase/oxidase by hypoxia in lung microvascular endothelial cells. Int J Biochem Cell Biol 40: 461-470, 2008.

39. Ladilov Y, Schäfer C, Held A, Schäfer M, Noll T and Piper HM: Mechanism of $\mathrm{Ca}(2+)$ overload in endothelial cells exposed to simulated ischemia. Cardiovasc Res 47: 394-403, 2000.

40. Makino J, Kamiya T, Hara $\mathrm{H}$ and Adachi T: TPA induces the expression of EC-SOD in human monocytic THP-1 cells: Involvement of PKC, MEK/ERK and NOX-derived ROS. Free Radic Res 46: 637-644, 2012.

41. Carbone F, Teixeira PC, Braunersreuther V,MachF, VuilleumierN and Montecucco F: Pathophysiology and treatments of oxidative injury in ischemic stroke: Focus on the phagocytic NADPH oxidase 2. Antioxid Redox Signal 23: 460-489, 2015.

42. Ansari MA and Scheff SW: NADPH-oxidase activation and cognition in Alzheimer disease progression. Free Radic Biol Med 51: 171-178, 2011.

43. Pearson G, Robinson F, Beers Gibson T, Xu BE, Karandikar M, Berman K and Cobb MH: Mitogen-activated protein (MAP) kinase pathways: Regulation and physiological functions. Endocr Rev 22: 153-183, 2001

44. Yuan L, Wang J, Xiao H, Wu W, Wang Y and Liu X: MAPK signaling pathways regulate mitochondrial-mediated apoptosis induced by isoorientin in human hepatoblastoma cancer cells. Food Chem Toxicol 53: 62-68, 2013.

45. Lin C, Holland RE Jr, Donofrio JC, McCoy MH, Tudor LR and Chambers TM: Caspase activation in equine influenza virus induced apoptotic cell death. Vet Microbiol 84: 357-365, 2002.

46. Vermes I, Haanen C and Reutelingsperger C: Flow cytometry of apoptotic cell death. J Immunol Methods 243: 167-190, 2000.

47. Muslin AJ: MAPK signalling in cardiovascular health and disease: Molecular mechanisms and therapeutic targets. Clin Sci (Lond) 115: 203-218, 2008.

48. Newhouse K, Hsuan SL, Chang SH, Cai B, Wang Y and Xia Z: Rotenone-induced apoptosis is mediated by $\mathrm{p} 38$ and JNK MAP kinases in human dopaminergic SH-SY5Y cells. Toxicol Sci 79: $137-146,2004$

This work is licensed under a Creative Commons Attribution-NonCommercial-NoDerivatives 4.0 International (CC BY-NC-ND 4.0) License. 